\title{
Separated and overlapping neural coding of face and body identity
}

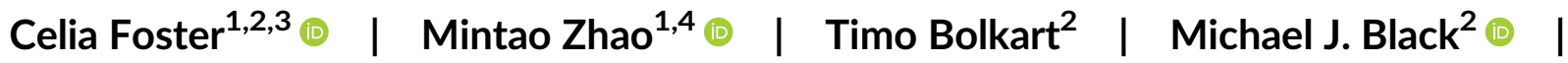 \\ Andreas Bartels ${ }^{1,5,6,7}$ @ | Isabelle Bülthoff ${ }^{1}$
}

${ }^{1}$ Max Planck Institute for Biological Cybernetics, Tübingen, Germany

${ }^{2}$ Max Planck Institute for Intelligent Systems, Tübingen, Germany

${ }^{3}$ International Max Planck Research School for Cognitive and Systems Neuroscience, University of Tübingen, Tübingen, Germany

${ }^{4}$ School of Psychology, University of East Anglia, UK

${ }^{5}$ Centre for Integrative Neuroscience, Tübingen, Germany

${ }^{6}$ Department of Psychology, University of Tübingen, Germany

${ }^{7}$ Bernstein Center for Computational Neuroscience, Tübingen, Germany

\section{Correspondence}

Celia Foster, Biopsychology \& Cognitive Neuroscience (AE14), Faculty of Psychology and Sports Science, Bielefeld University, POBox 100131, D-33501 Bielefeld, Germany. Email: celia.foster@uni-bielefeld.de

Isabelle Bülthoff, Max Planck Institute for Biological Cybernetics, Max-Planck-Ring 8, 72076 Tübingen, Germany.

Email: isabelle.buelthoff@tuebingen.mpg.de

\section{Present address}

Celia Foster, Biopsychology and Cognitive Neuroscience, Faculty of Psychology and Sports Science, Bielefeld University, Germany.

Funding information

Max-Planck-Gesellschaft

\begin{abstract}
Recognising a person's identity often relies on face and body information, and is tolerant to changes in low-level visual input (e.g., viewpoint changes). Previous studies have suggested that face identity is disentangled from low-level visual input in the anterior face-responsive regions. It remains unclear which regions disentangle body identity from variations in viewpoint, and whether face and body identity are encoded separately or combined into a coherent person identity representation. We trained participants to recognise three identities, and then recorded their brain activity using $\mathrm{fMRI}$ while they viewed face and body images of these three identities from different viewpoints. Participants' task was to respond to either the stimulus identity or viewpoint. We found consistent decoding of body identity across viewpoint in the fusiform body area, right anterior temporal cortex, middle frontal gyrus and right insula. This finding demonstrates a similar function of fusiform and anterior temporal cortex for bodies as has previously been shown for faces, suggesting these regions may play a general role in extracting high-level identity information. Moreover, we could decode identity across fMRI activity evoked by faces and bodies in the early visual cortex, right inferior occipital cortex, right parahippocampal cortex and right superior parietal cortex, revealing a distributed network that encodes person identity abstractly. Lastly, identity decoding was consistently better when participants attended to identity, indicating that attention to identity enhances its neural representation. These results offer new insights into how the brain develops an abstract neural coding of person identity, shared by faces and bodies.
\end{abstract}

KEYWORDS

body recognition, face recognition, FBA, identity, viewpoint

\section{1 | INTRODUCTION}

Being able to recognise the identity of people we encounter in our daily life is a crucial ability for our social interactions. We use multiple sources of information in order to encode and then subsequently recognise specific people, including facial information, body information, and face and body movements (Dobs, Bülthoff, \& Schultz, 2016; Hahn, O'Toole, \& Phillips, 2015; O'Toole et al., 2011; O'Toole,

This is an open access article under the terms of the Creative Commons Attribution-NonCommercial-NoDerivs License, which permits use and distribution in any medium, provided the original work is properly cited, the use is non-commercial and no modifications or adaptations are made.

(c) 2021 The Authors. Human Brain Mapping published by Wiley Periodicals LLC. 
Roark, \& Abdi, 2002; Rice, Phillips, Natu, An, \& O'Toole, 2013; Rice, Phillips, \& O'Toole, 2013; Robbins \& Coltheart, 2012; Simhi \& Yovel, 2016; Yovel \& O'Toole, 2016). For instance, our visual system is remarkably good at recognising the identity of familiar people, irrespective of changes in viewpoint, illumination, position, pose and expression. This seemingly effortless ability is computationally challenging, as these changes lead to a great variability in low-level visual information arriving on the retina, yet we are able to distinguish between identities that look comparably similar to one another. Although the face gives strong cues for recognition, body information also contributes to the visual recognition of a person's identity, especially when face information is not optimal for recognition, for example when a person is far away (Hahn et al., 2015; O'Toole et al., 2011; Rice, Phillips, Natu, et al., 2013; Rice, Phillips, \& O'Toole, 2013; Yovel \& O'Toole, 2016). It is not yet fully understood how person identity is encoded in the brain, and whether the neural coding of face identity and body identity is separated or overlapping. We aimed to address these questions in the present study.

Neural coding of face identity has been associated with faceresponsive brain regions in the fusiform gyrus and anterior temporal cortex (Haxby, Hoffman, \& Gobbini, 2000). These regions respond when participants recognise face identities (Grill-Spector, Knouf, \& Kanwisher, 2004; Hoffman \& Haxby, 2000; Nasr \& Tootell, 2012) and dysfunction of these regions can lead to impairments in face recognition ability (Barton, 2008; Busigny et al., 2014; Hadjikhani \& de Gelder, 2002; Jonas et al., 2015). Anterior temporal cortex is thought to be of particular importance in encoding high-level face identity representations. Patterns of activity in this region can distinguish between different face identities (Kriegeskorte, Formisano, Sorger, \& Goebel, 2007). Moreover, activity patterns evoked by different face identities in this region can generalise across face viewpoint (Anzellotti, Fairhall, \& Caramazza, 2014; Freiwald \& Tsao, 2010; Guntupalli, Wheeler, \& Gobbini, 2017; Natu et al., 2010), face expression (Nestor, Plaut, \& Behrmann, 2011) and different halves of the same face (Anzellotti \& Caramazza, 2016). The fusiform face area (FFA) also responds to changes in identity (Andrews \& Ewbank, 2004; Gauthier et al., 2000; Loffler, Yourganov, Wilkinson, \& Wilson, 2005; Rotshtein, Henson, Treves, Driver, \& Dolan, 2005; Winston, Henson, FineGoulden, \& Dolan, 2004), and some studies have shown that face identity responses in the FFA can generalise across viewpoint (Anzellotti et al., 2014; Guntupalli et al., 2017). Other studies have also found high-level face identity responses in the occipital face area (OFA) (Anzellotti et al., 2014), the superior intraparietal sulcus (Jeong \& Xu, 2016) and right inferior frontal cortex (Guntupalli et al., 2017).

Although psychological research has shown that we also use body information to recognise people (Hahn et al., 2015; O'Toole et al., 2011; Rice, Phillips, Natu, et al., 2013; Rice, Phillips, \& O'Toole, 2013; Robbins \& Coltheart, 2012; Simhi \& Yovel, 2016; Yovel \& O'Toole, 2016), much less is known about the brain regions encoding body identity. An fMRI repetition suppression study found lower responses in the extrastriate and fusiform body areas (EBA and FBA) to repeated presentation of the same body identity as compared to presentation of different body identities, suggesting that these regions encode body identity information (Ewbank et al., 2011). Stronger blood oxygen level-dependent (BOLD) responses to the bodies of familiar people, as compared to unfamiliar people, have been observed in the FBA as well as the inferior and medial frontal gyrus, cingulate gyrus, central and post-central sulcus and inferior parietal lobe (Hodzic, Kaas, Muckli, Stirn, \& Singer, 2009). It has also been shown that the familiarity vs. unfamiliarity of people viewed from a distance can be decoded from the EBA and FBA (Hahn \& O'Toole, 2017). However, it remains unknown which brain regions contain different patterns of BOLD responses evoked by different body identities, or furthermore which brain regions contain patterns of responses to different body identities that can generalise across different viewpoints. In macaques, electrophysiological recordings have shown that the body-responsive patches contain body identity information that can generalise across viewpoint and pose (Kumar, Popivanov, \& Vogels, 2019). Interestingly, identity decoding accuracy was higher in the more anterior body patch, suggesting an important role of more anterior temporal regions in encoding viewpoint-invariant body identity, similar to the function of more anterior face-responsive regions in viewpoint-invariant coding of face identity.

In the present study, we aimed to address two fundamental questions about the neural representation of face and body identity. Firstly, we investigated which brain regions encode body identity, and which regions encode body identity in a viewpoint-invariant manner. Secondly, we investigated whether face- and body-based identity information are encoded in separated or overlapping brain networks. Previous work has shown that the body-responsive EBA and FBA show stronger responses to whole bodies as compared to body parts, but show no difference in responses to whole faces compared to face parts, while the opposite pattern has been found for the face-responsive OFA and FFA (Brandman \& Yovel, 2016). Of particular interest is where in the brain face and body identity information from the same person is combined into a stimulus-independent person identity representation. It has been suggested that brain regions processing faces and bodies in occipitotemporal cortex are mostly separated, parallel networks (Pitcher, Charles, Devlin, Walsh, \& Duchaine, 2009; Premereur, Taubert, Janssen, Vogels, \& Vanduffel, 2016; Schwarzlose, Baker, \& Kanwisher, 2005). Different regions in these networks encode information about the familiarity vs. unfamiliarity of people depending on the distance, and thus the available face, body and gait information (Hahn \& O'Toole, 2017). Our recent study showed that certain aspects of face and body information (e.g., weight) are integrated in the EBA (Foster et al., 2019). In macaques, the anterior face patches show stronger neural responses to images of a whole person than to the addition of the responses to the face and body shown alone (Fisher \& Freiwald, 2015), suggesting that these regions may integrate face and body information. If face and body information is integrated to form an abstract neural person identity code, we would expect similar patterns of BOLD responses to a particular identity, regardless of whether the person is viewed from an image of their face or body.

To address these questions, we trained participants to recognise three identities and then recorded their brain activity using fMRI as 
they viewed images of the face and body of these three identities from three different viewpoints. Participants performed two behavioural tasks during the experiment, one where they responded to the stimulus identity (i.e., identity recognition task) and the other where they responded to the stimulus viewpoint (i.e., viewpoint recognition task). This manipulation allowed us to investigate if neural coding of person identity is enhanced when participants attend to identity as compared to when they do not (i.e., when they attend to viewpoint) First, to investigate which brain regions contained patterns of BOLD responses that could distinguish between face identities and between body identities, we trained linear support vector machine (SVM) classifiers to distinguish between patterns of activity evoked by the identities, and then tested these classifiers on their ability to decode face and body identities in a separate test set of data. Second, to test which brain regions contain patterns of brain activity that encode face or body identities in a viewpoint-invariant manner, we trained classifiers using BOLD responses evoked by the face/body identities from two viewpoints and then tested their ability to distinguish between BOLD responses evoked by the face/body identities from the third viewpoint (i.e., identity classifiers that could generalise across viewpoint). Third, to test which brain regions encoded person identity using an abstract code, independent of the stimulus type (i.e., faces or bodies), we trained a classifier using patterns of activity evoked by the face identities and then tested this classifier on its ability to distinguish between patterns of activity evoked by the body identities, and vice versa. We performed all of these analyses in face- and body-responsive regions of interest (ROIs) as well as in whole-brain searchlight analyses, and we performed these analyses separately on fMRI data where participants performed the identity and viewpoint recognition tasks.

\section{2 | MATERIALS AND METHODS}

\section{1 | Participants}

Twenty participants completed the experiment. One participant was excluded from the data analyses due to poor performance in the behavioural task ( $<40 \%$ correct responses in one condition). The remaining 19 participants ( 13 female, 6 male, $21-51$ years old) were included in the behavioural and fMRI analyses presented here. The experiment procedure was approved by the local ethics committee of the University Clinic Tübingen and was conducted according to the Declaration of Helsinki. All participants provided written informed consent prior to the start of the experiment.

\section{2 | Stimuli}

\subsection{1 | Main experiment stimuli}

Our stimuli (Figure 1a) consisted of separate face and body images of three identities from three viewpoints; $0^{\circ}$ (front), $45^{\circ}$ (three-quarter) and $90^{\circ}$ (profile). The three identities were all female, to ensure that sex did not differ between the three identities, and we selected identities that were easily visually differentiable from one another for both the face and body. For each identity, we recorded both a 3D face scan with a neutral expression and a 3D body scan in an A-pose. The face scans were then aligned to a 3D shape and expression model (Li, Bolkart, Black, Li, \& Romero, 2017) and the body scans were aligned to a 3D shape and pose model (Loper, Mahmood, Romero, Pons-Moll, \& Black, 2015). We then generated images of the three individuals from the three viewpoints $\left(0^{\circ}\right.$, $45^{\circ}$ and $90^{\circ}$ ). For body images, we covered the face using a grey rectangle to remove face information from the body images.

For each identity, we also recorded a short video showing the whole body with the head fully visible turning between the left and right profile view. This video was used for identity learning prior to the fMRI experiment (see procedure below).

\subsection{2 $\quad$ Localizer stimuli}

Stimuli used to localise face- and body-responsive regions of interests were grayscale images of faces, headless bodies, objects and phasescrambled images. The phase-scrambled images were generated by creating Fourier-scrambled versions of an image consisting of a collage of the face and headless body images.

\subsection{Experimental procedure}

The study consisted of a short identity learning session (outside of the MRI scanner) followed immediately by the main fMRI session, which consisted of eight runs of the main experiment and one run of a localizer experiment.

\subsection{1 | Identity learning}

We trained participants to recognise the three identities from images of their face and body. The identity learning session consisted of five repetitions of a learning and testing with feedback procedure. During learning, participants viewed a $15 \mathrm{~s}$ video of each identity (showing their whole body turning between the left and right profile), then viewed the separate face and body images of the identity from the three viewpoints $\left(0^{\circ}, 45^{\circ}\right.$ and $90^{\circ}$ ), until the participant pressed a button to continue. A name was presented above all the images of each identity, so that participants could learn to associate each identity with its name. Following learning, participants completed 54 trials of the testing procedure with feedback. The 54 trials consisted of three repetitions of the 18 stimulus conditions (face or body, three identities, three viewpoints) presented in a random order. In each trial, participants viewed a fixation cross for $1 \mathrm{~s}$, then a stimulus image for $1 \mathrm{~s}$, then a grey screen. Participants had up to $6 \mathrm{~s}$ to respond using a button press to indicate which identity was shown. After making a response, participants were given feedback as to whether their response was correct or not. At the end of the test, participants were shown an overall percentage correct score. The mean percentage correct score at the 
(a)

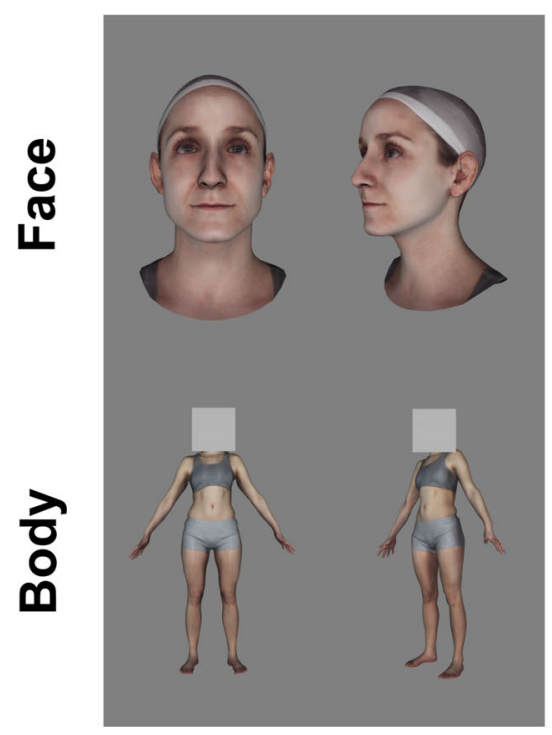

Identity 2

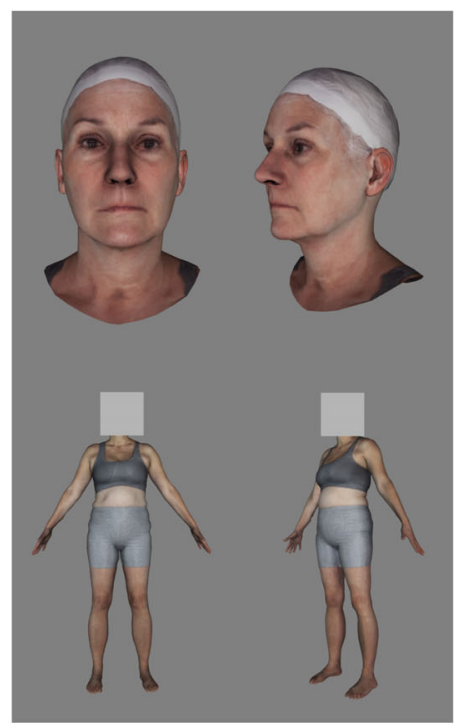

Identity 3

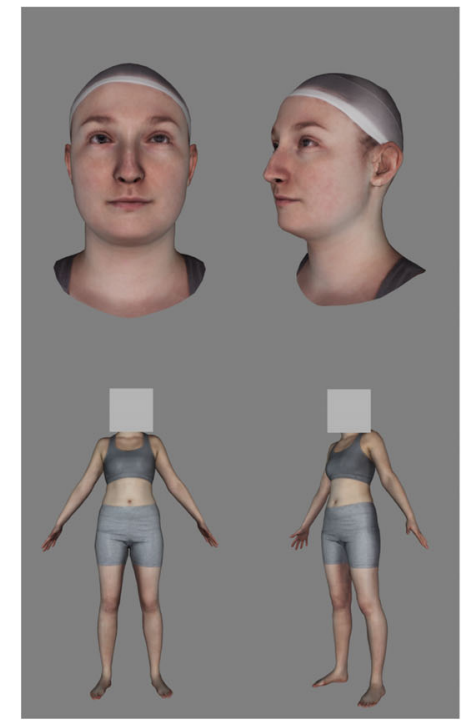

(b)

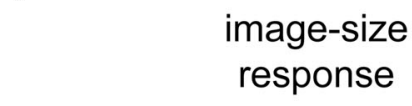
response

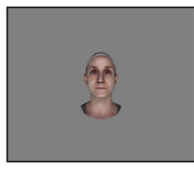

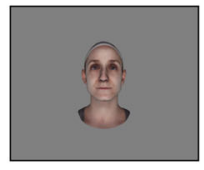

image-size response

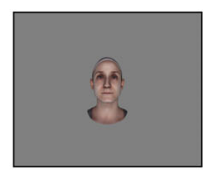

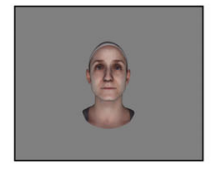

identity or viewpoint response

time

FIGURE 1 Experimental stimuli and procedure. (a) Stimuli were face and body images of three female identities shown from three viewpoints $\left(0^{\circ}\right.$ and $45^{\circ}$ shown here). (b) Example block of stimuli shown in the fMRI experiment. Participants viewed six images from one condition (i.e., face or body, one identity, one viewpoint) within a block, which varied in their image size (two repetitions of three image sizes, shown in a random order). Participants performed two tasks; they responded immediately when they saw an image of the smallest image size, and they responded at the end of the block during fixation to indicate which identity or viewpoint was shown in the block (half of the experiment trials were the identity recognition task, and the other half of the trials were the viewpoint recognition task)

end of the training session was $99.8 \%$ for faces and $97.3 \%$ for bodies. It is worth noting that here we refer 'identity' in its conventional meaning of 'who the person is' when we talk about person identity, face identity and body identity. We can recognise the identity of a person based on a variety of identity-specific characteristics (e.g., face, body shape, voice, gait, etc.), and here we specifically focused on the identity learned (and derived) solely from the visual information provided by the face or the body.

The identity learning session was presented on a laptop with resolution $1366 \times 768$, running Windows 10 with Matlab 2014a using the Psychophysics Toolbox extensions (Brainard, 1997; Kleiner, Brainard, \& Pelli, 2007; Pelli, 1997).

\subsection{2 | Main $\mathrm{fMRI}$ experiment}

Participants lay supine in the MRI scanner and viewed the stimuli on a screen positioned $92 \mathrm{~cm}$ behind their head, via a mirror attached to the head coil. We presented the stimuli in a block design, with each block showing images from 1 of 18 conditions of a 2 (face or body) $\times 3$ (identity) $\times 3$ (viewpoint) factorial design. Each run contained three repetitions of all 18 conditions presented in a random order. The 18 conditions were preceded by and followed by $8 \mathrm{~s}$ of fixation.

Each block contained six images varying in their image size (Figure 1b). There were two repetitions of three image sizes presented in a random order. The three image sizes had scale factors of $1,1.3$ and 1.6 (i.e., the largest image size was 1.6 times the width and height of the smallest image size). For face stimuli, the mean widths and heights of the three image sizes were $4.4^{\circ} \times 6.4^{\circ}, 3.6^{\circ} \times 5.2^{\circ}$ and $2.8^{\circ} \times 4.0^{\circ}$ of visual angle, for body stimuli the mean widths and heights of the 3 image sizes were $3.2^{\circ} \times 7.7^{\circ}, 2.6^{\circ} \times 6.2^{\circ}$ and $2.0^{\circ} \times 4.8^{\circ}$ of visual angle. Each image was shown for $900 \mathrm{~ms}$ and a $100 \mathrm{~ms}$ blank screen was shown between images. Each block was followed by $2 \mathrm{~s}$ fixation. 
The experiment was programmed with Matlab 2017a using the Psychophysics Toolbox extensions (Brainard, 1997; Kleiner et al., 2007) on Ubuntu 17.10. The experiment was presented using a projector with resolution $1920 \times 1080$ onto a screen with a width and height of $25^{\circ} \times 14^{\circ}$ of visual angle.

Participants performed an identity recognition task in half of the experiment runs and a viewpoint recognition task in the other half of the experiment runs. Each run began with an instruction informing the participant whether they should respond to the identity or the viewpoint of the stimuli in that run. Participants responded during fixation at the end of each block by pressing a corresponding button to indicate which identity (ID1, ID2 or ID3) or which viewpoint $\left(0^{\circ}, 45^{\circ}\right.$ or $90^{\circ}$ ) was shown in the block. To ensure participants kept their attention on the stimuli throughout each block, we instructed participants to immediately press a button with their thumb whenever they saw an image that was shown in the smallest of the three image sizes.

\subsection{3 | fMRI localizer experiment}

Participants completed one run of a localizer experiment which was used to define face- and body-responsive brain regions. Participants viewed face, body, object and phase-scrambled images in a block design. Each block consisted of eight images, which were each shown for $1.8 \mathrm{~s}$ followed by a $0.2 \mathrm{~s}$ blank screen. Blocks were presented in a carryover counterbalanced sequence, such that face, body, object and phase scrambled blocks were preceded by each other block type an equal number of times (Brooks, 2012). Face, body and object images were shown in front of the phase-scrambled images to keep the area of retinal stimulation the same for all blocks. Participants performed a one-back matching task on the images to keep their attention on the stimuli. Images were repeated on average once every $9 \mathrm{~s}$.

\section{$2.4 \quad$ MRI sequence parameters}

MRI data was acquired with a 3T Siemens Prisma scanner and a 64-channel head coil (Siemens, Erlangen, Germany). Functional T2* echoplanar images (EPI) were acquired using the following sequence parameters; multiband acceleration factor 2, GRAPPA acceleration factor 2, TR $1.84 \mathrm{~s}$, TE $30 \mathrm{~ms}$, flip angle 79 , FOV $192 \times 192 \mathrm{~mm}$. Volumes consisted of 60 slices and had an isotropic voxel size of $2 \times 2 \times 2 \mathrm{~mm}$. We discarded the first eight volumes of each run to allow for equilibration of the T1 signal. We additionally acquired a high-resolution T1-weighted anatomical scan for each participant with the following sequence parameters; TR $2 \mathrm{~s}$, TE $3.06 \mathrm{~ms}$, FOV $232 \times 256 \mathrm{~mm}, 192$ slices, isotropic voxel size of $1 \times 1 \times 1 \mathrm{~mm}$.

\section{5 | MRI data preprocessing}

We preprocessed our MRI data using SPM12 (http://www.fil.ion.ucl. ac.uk/spm/). Functional images were slice-time corrected, realigned and coregistered to the anatomical image. Functional images from the localizer experiment were additionally smoothed with a $6 \mathrm{~mm}$ Gaussian kernel. ROI and searchlight analyses on functional images from the main experiment were conducted on unsmoothed data in subjectspace. The resulting searchlight classification accuracy maps were then normalised to MNI (Montreal Neurological Institute) space, and spatially smoothed with a $6 \mathrm{~mm}$ Gaussian kernel. For the whole-brain univariate analyses the coregistered data was normalised to $\mathrm{MNI}$ space and spatially smoothed with a $6 \mathrm{~mm}$ Gaussian kernel.

\subsection{Definition of regions of interest}

Using $\mathrm{FMRI}$ data from the localizer experiment, we defined three faceresponsive ROIs (the OFA, FFA and ATFA) and two body-responsive ROls (the EBA and FBA), see Table 1. We first attempted to define the face-responsive ROls using the contrast faces > objects and the body-responsive ROIs using the contrast bodies > objects. If we could not define a ROI in a participant using this contrast, we then attempted to define the ROI using the contrast faces $>$ scrambled images or bodies > scrambled images. We initially used a contrast threshold of $p<.001$ (uncorrected) and reduced the threshold to $p<.01$ (uncorrected) if the ROI could not be defined with the initial threshold. For details on the number of participants for which each contrast and threshold was used see Tables S1 (face-responsive ROIs) and S2 (body-responsive ROls).

\section{7 | Behavioural analyses}

We calculated participants' accuracy in the identity and viewpoint recognition tasks using \% correct. To investigate if stimulus identity affected participants' ability to recognise the identity or viewpoint of the stimuli during the fMRI experiment, we performed one-way repeated-measures ANOVAs with three levels (ID1, ID2 and ID3), separately for identity and viewpoint recognition of the face and body stimuli. Prior to each ANOVA, we tested for non-sphericity using a Mauchly's test of sphericity, and where necessary we corrected for non-sphericity using a Greenhouse-Geisser correction.

\section{8 | Univariate $\mathrm{fMRI}$ analyses}

We conducted univariate analyses to investigate if there were any differences in the mean BOLD signal evoked by the three stimulus identities. To do this, we used SPM12 to model the fMRI data with a GLM. The GLM contained regressors for each of the experimental conditions. We then performed one-way repeated-measures ANOVAs with three levels (ID1, ID2 and ID3) separately for face and body stimuli, in face- and body-responsive ROIs and in whole-brain analyses. For ROI analyses, we tested for non-sphericity using a Mauchly's test of sphericity, and where necessary corrected for non-sphericity using a Greenhouse-Geisser correction. We then assessed significance 
TABLE 1 Mean MNI coordinates and volume of each ROI, $\pm S D$. $N$ shows the number of participants each ROI was identified in

\begin{tabular}{rlrcrrr|}
\hline ROI & Hem & \multicolumn{1}{c}{$\boldsymbol{x}$} & $\boldsymbol{y}$ & $\boldsymbol{z}$ & Volume $\left(\mathrm{mm}^{3}\right)$ & $\boldsymbol{N}$ \\
\hline OFA & Left & $-35 \pm 6.9$ & $-86 \pm 5.9$ & $-11 \pm 3.6$ & $731 \pm 346.5$ & 19 \\
& Right & $38 \pm 4.1$ & $-81 \pm 6.0$ & $-10 \pm 3.3$ & $994 \pm 382.8$ & 19 \\
\hline FFA & Left & $-40 \pm 2.8$ & $-55 \pm 5.5$ & $-20 \pm 2.8$ & $709 \pm 364.3$ & 19 \\
& Right & $42 \pm 3.3$ & $-52 \pm 4.3$ & $-18 \pm 2.4$ & $1,083 \pm 400.9$ & 19 \\
\hline \multirow{2}{*}{ ATFA } & Left & $-34 \pm 5.5$ & $-11 \pm 6.7$ & $-33 \pm 6.9$ & $177 \pm 120.6$ & 14 \\
& Right & $34 \pm 5.8$ & $-8 \pm 5.5$ & $-37 \pm 5.8$ & $335 \pm 265.6$ & 18 \\
\hline \multirow{2}{*}{ EBA } & Left & $-44 \pm 3.7$ & $-78 \pm 5.4$ & $3 \pm 6.6$ & $896 \pm 486.0$ & 19 \\
& Right & $49 \pm 2.3$ & $-70 \pm 2.7$ & $0 \pm 4.7$ & $1,686 \pm 453.0$ & 19 \\
\hline FBA & Left & $-39 \pm 4.2$ & $-50 \pm 6.5$ & $-20 \pm 3.0$ & $703 \pm 459.0$ & 18 \\
& Right & $40 \pm 3.9$ & $-50 \pm 5.6$ & $-19 \pm 2.4$ & $1,148 \pm 552.6$ & 19 \\
\hline
\end{tabular}

using a threshold of $p<.05$, Bonferroni-corrected for $N=5$ ROls. Following any significant ANOVA results, we performed follow-up paired $t$-tests between the three identities to determine between which identities there were differences in BOLD responses. For whole-brain analyses, we assessed significance using a threshold of $p<.05$, false discovery rate (FDR) corrected.

\section{9 | Multivoxel pattern analyses (MVPA)}

We conducted multivoxel pattern analyses (MVPA) to investigate if there were differences in the patterns of BOLD responses evoked by the three stimulus identities. To do this, we first used SPM12 to model the fMRI data with a GLM. This GLM contained one regressor for each stimulus block. We then performed MVPA analyses on the beta weight images from the GLM using The Decoding Toolbox (Hebart, Görgen, \& Haynes, 2015). We feature-scaled the data using $z$-score normalisation, where we estimated the mean and SD across all conditions of the training data and applied these values to the test data. Any outlier values (greater than 2 SD from the mean) were set to 2 or -2 . This feature-scaling was conducted to bring the data into an optimal range for the classifier (Hebart et al., 2015). We performed three different classification analyses using a linear SVM classifier (LIBSVM).

We performed all classification analyses in face- and bodyresponsive brain regions and in whole-brain searchlight analyses (4-voxel radius). For ROI analyses, significance was determined using permutation testing. Each analysis was repeated 10,000 times with the condition labels randomly assigned to generate a null distribution of mean classification accuracies expected by chance. We assessed significance by comparing how often we obtained a mean classification accuracy in the null distribution greater than or equal to the actual mean classification accuracy obtained for that ROI. We assessed significance using a threshold of $p<.05$, and used a Bonferroni-correction for $N=5$ ROls tested. Additionally, we performed 5 (ROI) $\times 2$ (Task) repeated measures ANOVAs to test for significant differences in decoding performance across ROIs and the two recognition tasks, and we performed follow-up paired $t$-tests to investigate any significant main effects or interactions in these ANOVAs.
For searchlight analyses, we performed group analyses using nonparametric permutation tests with SnPM13 (http://warwick.ac.uk/ snpm). We performed 10,000 permutations for each analysis and used $6 \mathrm{~mm}$ FWHM variance smoothing. We assessed significance with a threshold of $p<.05$, FDR corrected.

\subsection{1 | Identity classification analyses}

We performed identity classification analyses to investigate which brain regions contain different patterns of activity evoked by different identities. We performed these analyses separately for BOLD responses evoked by face and body stimuli, and when participants performed the identity and viewpoint recognition tasks. We trained a linear SVM classifier to distinguish between patterns of BOLD responses evoked by the three identities using three runs of $\mathrm{fMRI}$ data. We then tested the classifier on its ability to predict the stimulus identities from BOLD responses in the fourth run of data. We performed a four-fold crossvalidation procedure (where each run was used as the held-out test dataset once), and we determined the final decoding accuracy by averaging over the four cross-validation iterations.

\subsection{2 | Viewpoint-invariant identity classification analyses}

We performed viewpoint-invariant identity classification analyses to investigate which brain regions contain patterns of BOLD responses evoked by the stimulus identities that can generalise across stimulus viewpoint. As previously, we performed these analyses separately for BOLD responses evoked by face and body stimuli, and when participants performed the identity and viewpoint recognition tasks. In these viewpoint-invariant analyses, we used three runs of fMRI data to train a linear SVM classifier to distinguish between patterns of BOLD responses evoked by the three identities from two of three viewpoints. We then tested the classifier on its ability to predict the stimulus identities from BOLD responses evoked by the third viewpoint in the fourth run of data. Again, we performed a four-fold crossvalidation procedure, and also repeated the analysis three times with 
each viewpoint used as the held out test viewpoint once. We determined the final decoding accuracy by averaging over the four crossvalidation iterations and the three viewpoint training and testing combinations.

\subsection{3 | Identity classification across face and body stimuli}

We investigated which regions contain patterns of activity evoked by the stimulus identities that can generalise across activity evoked by faces and bodies. We performed these classification analyses separately for BOLD responses while participants performed the identity and viewpoint recognition tasks. We trained a linear SVM classifier to distinguish between patterns of BOLD responses evoked by the three face identities using three runs of $\mathrm{fMRI}$ data. We then tested the classifier on its ability to predict the identity of the body stimuli in the fourth run of data. We performed a four-fold cross-validation procedure, and also repeated the analysis using BOLD responses evoked by bodies for training the classifier and BOLD responses evoked by faces for testing it. We determined the final decoding accuracy by averaging over the four cross-validation iterations and the two training and test set combinations.

\section{3 | RESULTS}

\section{1 | Behavioural results}

\subsection{1 | Identity recognition}

Participants' performance in the identity recognition task was high for both face (96.2\%) and body (93.7\%) stimuli (Figure 2a,c). We investigated if there were any differences in our participants' ability to recognise the three identities. One-way repeated measures ANOVAs with three levels (ID1, ID2 and ID3) showed that there were no significant differences in participants' ability to recognise the three identities from the face $\left(F_{2,36}=0.53, p=.59, \eta_{p}{ }^{2}=0.029\right)$ or body $\left(F_{2,36}=1.20, p=.31, \eta_{p}{ }^{2}=0.063\right)$ stimuli. These results show that participants could easily recognise all stimuli identities from both the faces and bodies.

\subsection{2 | Viewpoint recognition}

Participants showed high viewpoint recognition performance for both face (94.0\%) and body (94.6\%) stimuli (Figure 2b,d). One-way repeated measures ANOVAs with three levels (ID1, ID2 and ID3) revealed no significant effect of identity on participants' ability to recognise the viewpoint of faces $\left(F_{2,36}=2.04, p=.14, \eta_{p}{ }^{2}=0.10\right)$ or bodies $\left(F_{2,36}=0.18, p=.84, \eta_{p}{ }^{2}=0.010\right)$. Therefore, participants could recognise the stimulus viewpoints equally well regardless of the stimulus identity.

\section{2 $\quad$ Univariate fMRI results}

To investigate whether the three identities evoked different mean levels of BOLD activity, we performed one-way repeated measures ANOVAs with three levels (ID1, ID2 and ID3) in face- and bodyresponsive ROIs and in whole-brain analyses. The results are shown in Figure 3.

\subsection{1 | Face identity responses}

We performed one-way repeated measures ANOVAs with three levels (ID1, ID2 and ID3) to test whether there were any differences in the mean BOLD activity evoked by the three face identities. Full results of these ANOVAs in our ROls are shown in Table 2. For the identity recognition task, we found no significant differences between the mean BOLD activity evoked by the three face identities in any of our face- or body-responsive ROls (Figure 3a) or in any other region in a whole-brain analysis. For the viewpoint recognition task (Figure 3b), we (a) Face Identity Recognition

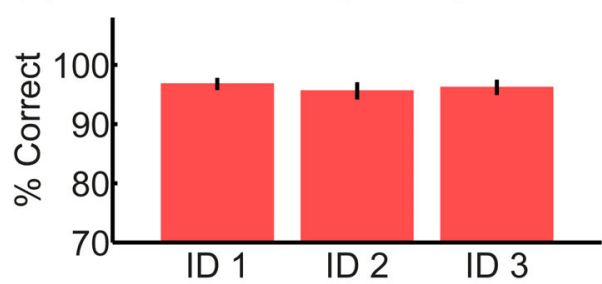

(c)

Body Identity Recognition

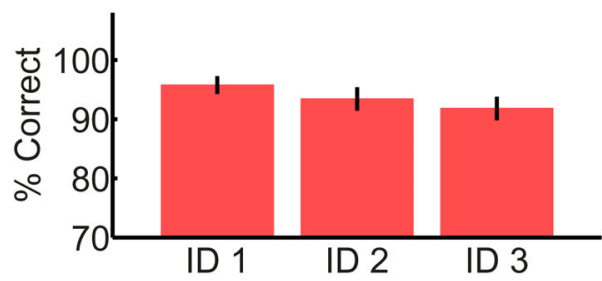

(b) Face Viewpoint Recognition

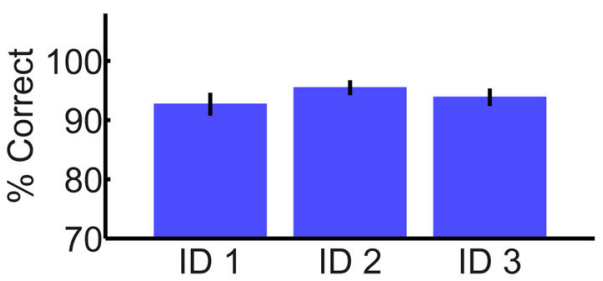

(d)

Body Viewpoint Recognition

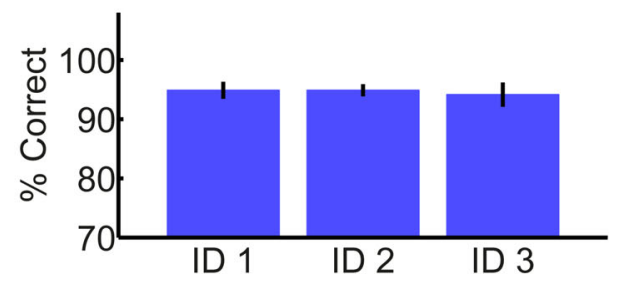

FIGURE 2 Recognition of the identity and viewpoint of the three stimulus identities (ID1, ID2 \& ID3). Panels (a) and (c) show identity recognition accuracy (\% correct) for the three stimulus identities from the face (a) and body (c) images. Panels (b) and (d) show viewpoint recognition accuracy (\% correct) for the three stimulus identities from the face (b) and body (d) images. Error bars indicate \pm 1 SEM 


\section{Identity recognition task}

(a)

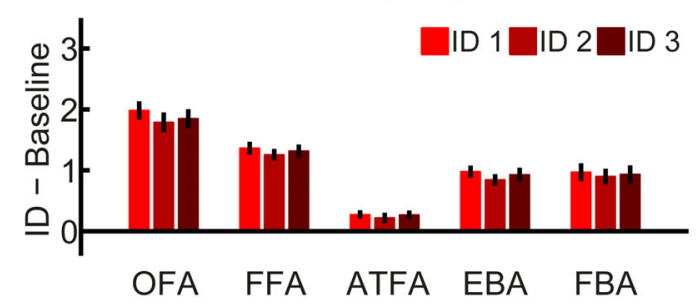

(c)

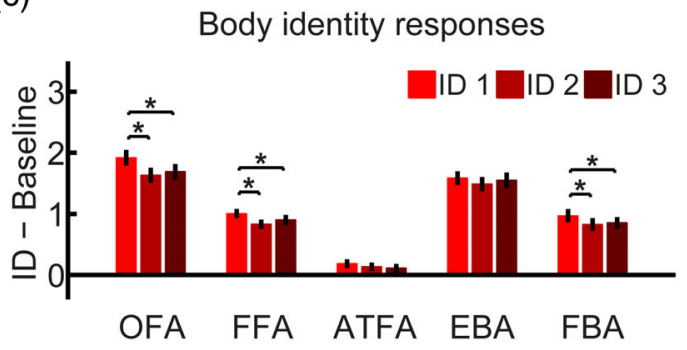

(e)

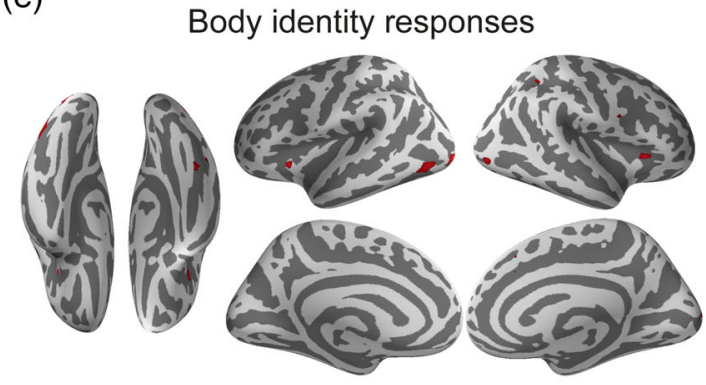

$F(2,36)$
Face identity responses

(b)

\section{Viewpoint recognition task}

Face identity responses

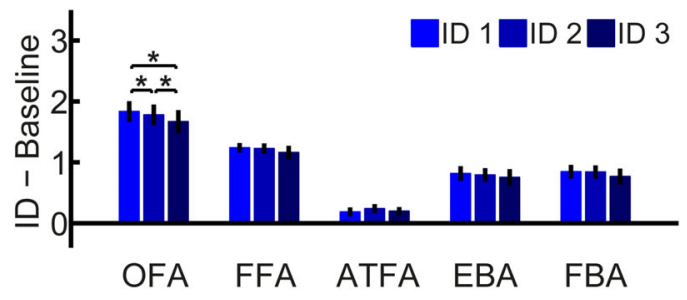

(d)

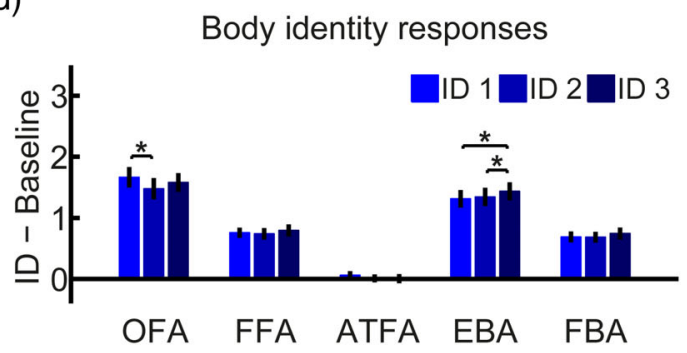

(f)

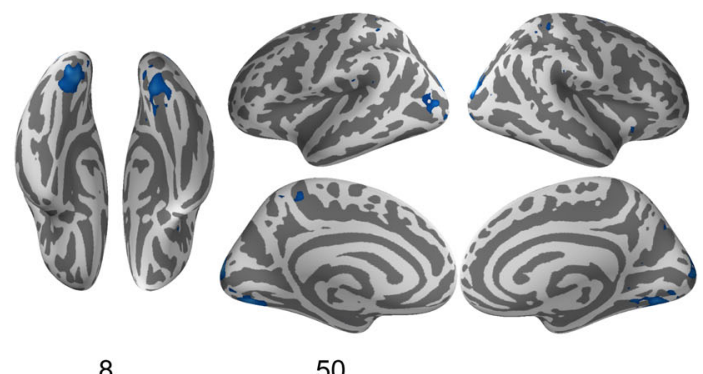

$F(2,36)$

FIGURE 3 Differences in mean BOLD response to the three identities. Panels (a) and (b) show mean BOLD responses to the three face identities in face- and body-responsive ROls during the identity (a) and viewpoint (b) recognition task. Panels (c) and (d) show mean BOLD responses to the three body identities in face- and body-responsive ROls during the identity (c) and viewpoint (d) recognition task. Panels (e) and (f) show differences in mean BOLD responses to the three body identities in whole-brain analyses (FDR corrected) during the identity (e) and viewpoint (f) recognition task. ${ }^{*}$ indicates $p<.05$

TABLE 2 Results of one-way repeated measures ANOVAs testing the effect of face identity on mean BOLD responses in face and bodyresponsive ROIs

\begin{tabular}{|llccll|}
\hline Behavioural task & ROI & $\boldsymbol{F}_{2,36}$ & $\boldsymbol{p}$ (corrected) & $\boldsymbol{p}$ (uncorrected) & $\boldsymbol{\eta}_{\boldsymbol{p}}{ }^{2}$ \\
\hline Identity recognition task & OFA & 6.53 & .056 & .011 & 0.27 \\
& FFA & 3.52 & .201 & .040 & 0.16 \\
& ATFA & 1.52 & 1.00 & .234 & 0.08 \\
& EBA & 4.51 & .089 & .018 & 0.20 \\
& FBA & 1.71 & .977 & .196 & 0.09 \\
\hline Viewpoint recognition task & OFA & 10.27 & .008 & .002 & 0.36 \\
& FFA & 2.47 & .594 & .119 & 0.12 \\
& ATFA & 0.99 & 1.00 & .382 & 0.05 \\
& EBA & 1.55 & 1.00 & .227 & 0.08 \\
& FBA & 2.70 & .484 & .097 & 0.13 \\
\hline
\end{tabular}

Note: $p$ (corrected) represents $p$ values Bonferroni-corrected for multiple comparisons ( $N=5 \mathrm{ROIs}$ ). All $p$ values are Greenhouse-Geisser corrected for any cases of non-sphericity. 
found significant differences between the mean BOLD activity evoked by the three face identities in the OFA $\left(F_{2,36}=10.27, p=.0075\right)$ but not in any other ROls. Follow-up paired $t$-tests showed that in the OFA there was higher activity to ID1 compared to ID2 $(M=0.056, S E=0.023$, $t_{18}=2.43, p=.026$, Cohen's $\left.d=0.56\right)$ and ID3 ( $M=0.17, S E=0.042$, $t_{18}=4.03, p<.001$, Cohen's $d=0.93$ ), and higher activity to ID2 than ID3 ( $M=0.11, S E=0.045, t_{18}=2.50, p=.023$, Cohen's $d=0.57$ ). In addition, a whole-brain analysis identified small, bilateral clusters in the early visual cortex showing differences in BOLD activity to the three face identities during the viewpoint recognition task.

\subsection{2 | Body identity responses}

We performed the same analysis on the BOLD responses evoked by body identities. ROI results are shown in Table 3 . For the identity recognition task (Figure 3c), we found significant differences in the mean BOLD responses evoked by the three body identities in the FBA $\left(F_{2,36}=6.96, p=.014\right)$, OFA $\left(F_{2,36}=20.76, p<.001\right)$ and FFA $\left(F_{2,36}=11.21, p<.001\right)$, but not in the EBA or ATFA. Follow-up paired $t$-tests revealed higher activity to ID1 compared to ID2 (FBA: $M=0.14, S E=0.028, t_{18}=5.03, p<.001$, Cohen's $d=1.15$; OFA: $M=0.29, S E=0.044, t_{18}=6.56, p<.001$, Cohen's $d=1.50$; FFA: $M=0.17, S E=0.030, t_{18}=5.67, p<.001$, Cohen's $\left.d=1.30\right)$ and ID3 (FBA: $M=0.11, S E=0.047, t_{18}=2.40, p=.027$, Cohen's $d=0.55$; OFA: $M=0.23, S E=0.046, t_{18}=4.98, p<.001$, Cohen's $d=1.14$; FFA: $M=0.10, S E=0.038, t_{18}=2.67, p=.016$, Cohen's $d=0.61$ ) but no difference between activity to ID2 and ID3 (FBA: $M=-0.028, S E=0.043, t_{18}=-0.66, p=.52$, Cohen's $d=-0.15$; OFA: $M=-0.058, S E=0.051, t_{18}=-1.13, p=.27$, Cohen's $d=-0.26$; FFA: $M=-0.071, S E=0.041, t_{18}=-1.74$, $p=.098$, Cohen's $d=-0.40$ ). We performed a whole-brain analysis to investigate if there were any additional regions showing different levels of mean BOLD activity to the three body identities during the identity recognition task (Figure $3 e$ ). We identified bilateral clusters in the early visual cortex, occipitotemporal cortex (overlapping with the locations of the OFA, FFA and FBA) and insula cortex, and unilateral clusters in the right inferior parietal cortex, right precuneus and right medial superior frontal gyrus.

For the viewpoint recognition task (Figure 3d), we found significant differences in activity evoked by the three body identities in the OFA $\left(F_{2,36}=6.52, p=.019\right)$ and EBA $\left(F_{2,36}=6.11, p=.026\right)$, but not in any other ROls. Follow-up paired $t$-tests showed lower activity in the OFA to ID2 compared to ID1 $(M=-0.19$, SE $=0.053$, $t_{18}=-3.55, p=.0023$, Cohen's $d=-0.81$ ) and higher activity in the EBA to ID3 compared to both ID1 ( $M=0.12, S E=0.036, t_{18}=3.43$, $p=.0030$, Cohen's $d=0.79)$ and ID2 (M=0.091, SE $=0.036$, $t_{18}=2.51, p=.022$, Cohen's $d=0.58$ ). We performed a whole-brain analysis to investigate if any other regions would show different levels of mean response to the three body identities during the viewpoint recognition task. We identified bilateral clusters in the early visual cortex, middle occipital cortex, fusiform gyrus, superior temporal cortex, superior parietal cortex, precuneus, superior frontal cortex and insula cortex (Figure 3f).

\section{3 | Face identity MVPA}

To investigate which brain regions contain separable patterns of BOLD responses for individual face identities, we performed multivoxel pattern analyses in face- and body-responsive ROls and in whole-brain searchlight analyses. Specifically, we first investigated whether face identity could be classified from patterns of BOLD responses, and secondly, whether identity classification could generalise across viewpoints. The results are shown in Figure 4.

\subsection{1 | Face identity classification}

We first performed ROI-based MVPA to investigate which face- and body responsive ROIs could decode face identity above chance-level (i.e., 1/3; see Section 2.9.1 for details of methods). The results are shown in Figure 4a (see Table 4 for full results). From the identity recognition task data, we were able to decode face identity significantly

\begin{tabular}{|llllll|}
\hline Behavioural task & ROI & $\boldsymbol{F}_{2,36}$ & $\boldsymbol{p}$ (corrected) & $\boldsymbol{p}$ (uncorrected) & $\boldsymbol{\eta}_{\boldsymbol{p}}{ }^{2}$ \\
\hline Identity recognition task & OFA & 20.76 & $<.001$ & $<.001$ & 0.54 \\
& FFA & 11.21 & $<.001$ & $<.001$ & 0.38 \\
& ATFA & 1.75 & .942 & .188 & 0.09 \\
& EBA & 2.02 & .740 & .148 & 0.10 \\
& FBA & 6.96 & .014 & .003 & 0.28 \\
\hline Viewpoint recognition task & OFA & 6.52 & .019 & .004 & 0.27 \\
& FFA & 1.43 & 1.00 & .253 & 0.07 \\
& ATFA & 2.27 & .588 & .118 & 0.11 \\
& EBA & 6.11 & .026 & .005 & 0.25 \\
& FBA & 1.88 & .834 & .167 & 0.10 \\
\hline
\end{tabular}

TABLE 3 Results of one-way repeated measures ANOVAs testing the effect of body identity on mean BOLD responses in face and bodyresponsive ROls

Note: $p$ (corrected) represents $p$ values Bonferroni-corrected for multiple comparisons ( $N=5$ ROIs). All $p$ values are Greenhouse-Geisser corrected for any cases of non-sphericity. 


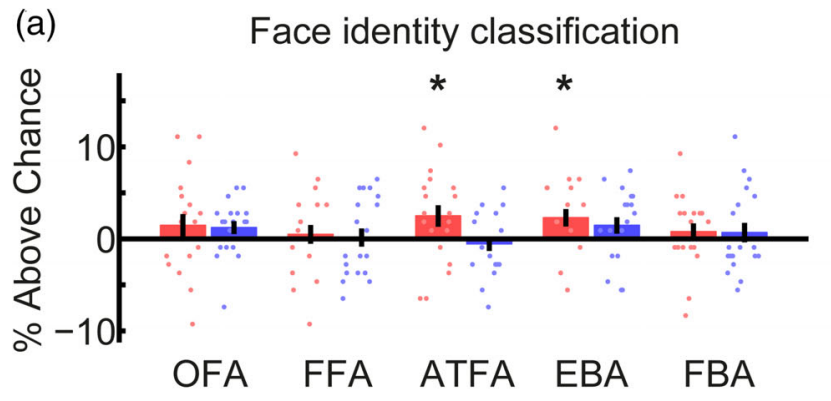

identity recognition task $\square$ viewpoint recognition task

(b)

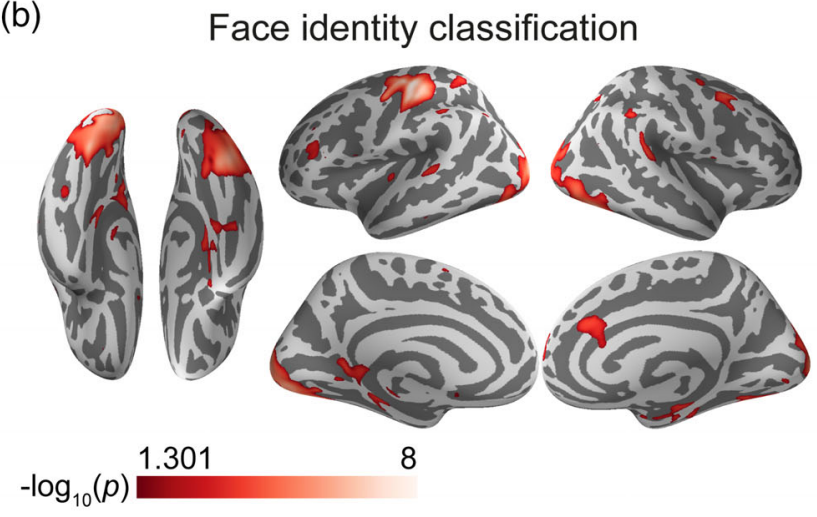

(c)

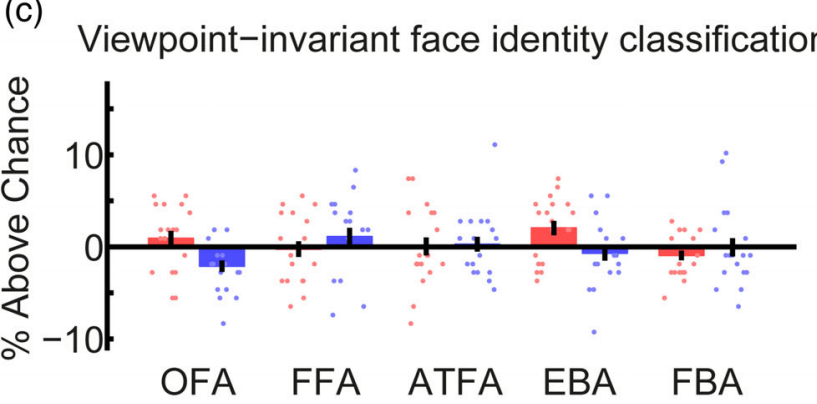

identity recognition task viewpoint recognition task of ROI $\left(F_{4,72}=0.68, p=.608, \eta_{p}^{2}=0.04\right)$ or Task $\left(F_{1,18}=1.91\right.$, $\left.p=.184, \eta_{p}{ }^{2}=0.10\right)$ or an interaction between the two factors $\left(F_{4,72}=0.91, p=.466, \eta_{p}{ }^{2}=0.05\right)$, suggesting that face identity decoding performance did not vary significantly across ROls or recognition task.

Secondly, we performed a whole-brain searchlight analysis to investigate if any other brain regions could decode face identity. Using fMRI data from the identity recognition task we identified clusters than could decode face identity bilaterally in the early visual cortex, inferior occipital cortex, fusiform gyrus, superior parietal cortex, superior temporal cortex and parahippocampal gyrus, and unilaterally in the right middle frontal gyrus, right anterior cingulum, right medial superior frontal gyrus and left inferior frontal gyrus (Figure 4b). We could also decode identity in the left motor cortex as participants pressed different buttons for each stimulus identity. Using fMRI data from the viewpoint recognition task, we were unable to decode face identity from any regions. We additionally performed a paired $t$-test to investigate whether any regions showed significant differences in decoding performance during the two recognition tasks. We did not identify any regions in this analysis.

\subsection{2 | Viewpoint-invariant face identity classification}

We next investigated which regions could classify face identity across viewpoint using the methods described in Section 2.9.2. Results of this analysis in face- and body-responsive ROls are summarised in Table 4. We were unable to decode face identity across viewpoint from any of the ROIs we tested (Figure 4c) using fMRI data from either the identity recognition task or the viewpoint recognition task. We performed searchlight analyses to investigate if any other brain regions would be able to decode face identity across viewpoint. We did not identify any regions in these analyses.

\section{4 | Body identity MVPA}

We performed the same MVPA analyses for bodies as for faces, to investigate which brain regions contain separable patterns of BOLD responses evoked by different body identities, and to investigate whether the neural coding of body identity can generalise across different viewpoints. We performed these analyses in face- and bodyresponsive ROIs and in whole-brain searchlight analyses.

\subsection{1 | Body identity classification}

body-responsive EBA (35.6\%, $p=.045$ ), but not from other faceresponsive ROIs or the FBA. From the viewpoint recognition task data, we were not able to decode face identity from any ROls. To investigate whether there were significant differences in decoding performance across ROls and Task, we performed a 5 (ROI) $\times 2$ (Task) repeated measures ANOVA. We did not find a significant main effect
First, we investigated which of our face- and body-responsive ROls could decode body identity above chance-level (1/3; see Section 2.9.1 for method details). The results are shown in Figure 5 a (see Table 5 for full results of ROI-based classification of body identity). From the identity recognition task, we could decode body identity significantly 
TAB LE 4 Classification of face identity from patterns of BOLD responses in individual ROls

\begin{tabular}{|c|c|c|c|c|c|c|}
\hline Analysis & Behavioural task & ROI & $\begin{array}{l}\text { Classification } \\
\text { accuracy (\%) }\end{array}$ & $\begin{array}{l}p \\
\text { (corr.) }\end{array}$ & $\begin{array}{l}p \\
\text { (uncorr.) }\end{array}$ & Cohen's $d$ \\
\hline \multirow[t]{8}{*}{ Face identity classification } & \multirow[t]{3}{*}{ Identity recognition task } & OFA & 34.8 & .318 & .064 & 0.27 \\
\hline & & ATFA & 35.8 & .031 & .006 & 0.49 \\
\hline & & EBA & 35.6 & .045 & .009 & 0.56 \\
\hline & \multirow{5}{*}{$\begin{array}{l}\text { Viewpoint recognition } \\
\text { task }\end{array}$} & OFA & 34.6 & .505 & .101 & 0.40 \\
\hline & & FFA & 33.5 & 1.00 & .443 & 0.03 \\
\hline & & ATFA & 32.8 & 1.00 & .705 & -0.16 \\
\hline & & EBA & 34.8 & .335 & .067 & 0.37 \\
\hline & & FBA & 34.0 & 1.00 & .224 & 0.15 \\
\hline \multirow{7}{*}{$\begin{array}{l}\text { Viewpoint-invariant face identity } \\
\text { classification }\end{array}$} & \multirow{2}{*}{ Identity recognition task } & EBA & 35.4 & .059 & .012 & 0.60 \\
\hline & & FBA & 32.4 & 1.00 & .862 & -0.40 \\
\hline & \multirow{5}{*}{$\begin{array}{l}\text { Viewpoint recognition } \\
\text { task }\end{array}$} & OFA & 31.2 & 1.00 & .991 & -0.77 \\
\hline & & FFA & 34.5 & .551 & .110 & 0.27 \\
\hline & & ATFA & 33.6 & 1.00 & .373 & 0.08 \\
\hline & & EBA & 32.7 & 1.00 & .773 & -0.19 \\
\hline & & FBA & 33.3 & 1.00 & .513 & -0.01 \\
\hline
\end{tabular}

Note: Statistical significance was assessed using permutation tests, and $p$ values are shown before (uncorrected) and after (corrected) Bonferronicorrection for multiple comparisons ( $N=5$ ROIs).

above chance from the body-responsive FBA $(36.4 \%, p=.0045)$ and face-responsive OFA $(38.5 \%, p<.001)$, but not from the bodyresponsive EBA or any other ROls. From the viewpoint recognition task, we were able to decode body identity from the OFA $(40.7 \%$, $p<.001$ ), but not from any other ROls.

To investigate whether there were significant differences in decoding performance across ROls and Task, we performed a 5 (ROI) $\times 2$ (Task) repeated measures ANOVA. We found a significant main effect of ROI $\left(F_{4,72}=18.09, p<.001, \eta_{p}{ }^{2}=0.50\right)$, as well as a significant interaction between $\mathrm{ROI}$ and Task $\left(F_{4,72}=4.45, p=.003\right.$, $\eta_{p}{ }^{2}=0.20$ ). Follow-up paired $t$-tests showed that decoding performance was significantly higher in the OFA compared to the FFA, ATFA and EBA (OFA \& FFA: $t_{18}=2.73, p=.014$, Cohen's $d=0.63$; OFA \& ATFA: $t_{18}=5.41, p<.001$, Cohen's $d=1.24$; OFA \& EBA: $t_{18}=3.00, p=.008$, Cohen's $d=0.69$ ) during the identity recognition task, and significantly higher than all ROls during the viewpoint recognition task (OFA \& FFA: $t_{18}=5.55, p<.001$, Cohen's $d=1.27$; OFA \& ATFA: $t_{18}=5.15, p<.001$, Cohen's $d=1.18$; OFA \& EBA: $t_{18}=5.38, p<.001$, Cohen's $d=1.23$; OFA \& FBA: $t_{18}=7.81$, $p<.001$, Cohen's $d=1.79$ ). Furthermore, decoding performance was significantly higher in the FFA, EBA and FBA compared to the ATFA during the identity recognition task (FFA \& ATFA: $t_{18}=2.91$, $p=.009$, Cohen's $d=0.67$; EBA \& ATFA: $t_{18}=3.47, p=.003$, Cohen's $d=0.80$; FBA \& ATFA: $t_{18}=2.95, p=.009$, Cohen's $d=0.68$ ). Decoding performance was significantly higher during the viewpoint recognition task than the identity recognition task in the ATFA ( $t_{18}=2.22, p=.039$, Cohen's $d=0.51$ ), but there were no significant differences between decoding performance due to task in any other ROIs.

Next, we performed a whole-brain searchlight analysis to investigate if we could decode body identity from any other brain regions. As illustrated in Figure 6a, we could decode body identity from a large area of occipital cortex using fMRI data from both the identity and viewpoint recognition tasks. Using fMRI data from the identity recognition task, we could also decode body identity from bilateral regions in the fusiform gyrus, superior parietal cortex, inferior frontal gyrus and middle frontal gyrus, and unilaterally from the right anterior temporal cortex and right insula cortex. We could also decode body identity in the left motor cortex as participants pressed different buttons to indicate the stimulus identity. Using fMRI data from the viewpoint recognition task, we could also decode body identity from bilateral regions in the fusiform gyrus, superior parietal cortex, supramarginal gyrus, cingulum, precentral gyrus and the caudate nucleus, and unilaterally from the right superior frontal gyrus. We additionally performed a paired $t$-test to investigate whether any regions showed significant differences in decoding performance during the two recognition tasks, but we did not identify any regions in this analysis. 
(a)

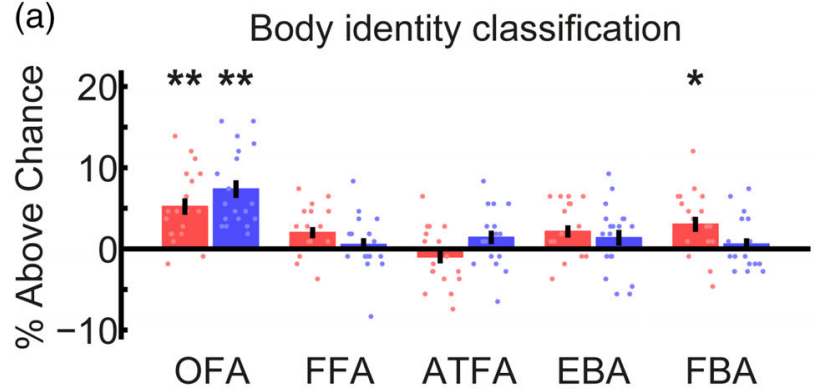

identity recognition task $\square$ viewpoint recognition task

(b)

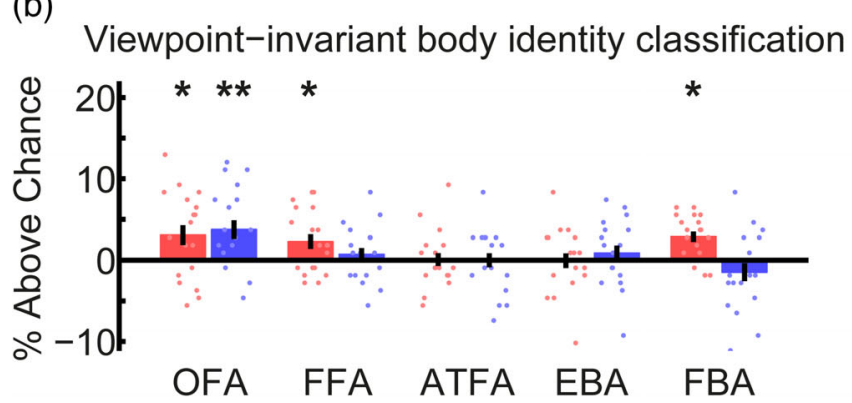

identity recognition task $\square$ viewpoint recognition task (c)
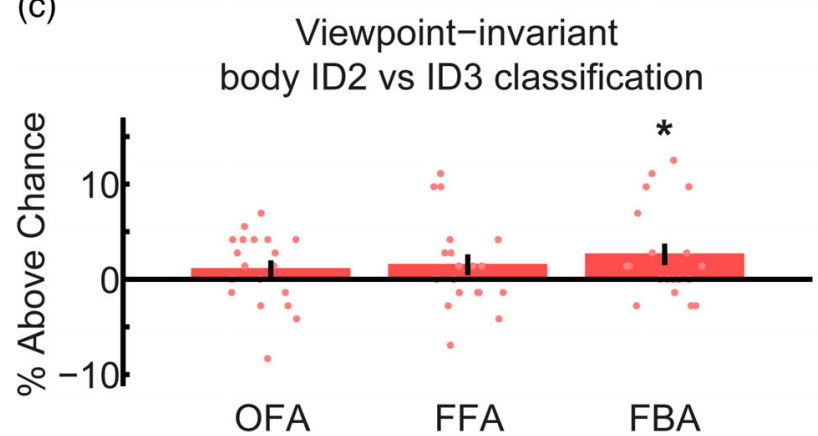

FIGURE 5 Classification and viewpoint-invariant classification of body identity in face- and body-responsive ROls. Panel (a) shows body identity classification above chance-level (1/3), panel (b) shows viewpoint-invariant body identity classification above chance-level (1/3) and panel (c) shows viewpoint-invariant body identity classification above chance-level (1/2) for ID2 versus ID3 only, with fMRI data from the identity recognition task. Scatter points show classification accuracies for individual participants and error bars show \pm 1 SEM. ${ }^{* *}$ indicates $p<.001,{ }^{*}$ indicates $p<.05$, Bonferroni corrected

\subsection{2 | Viewpoint-invariant body identity classification}

We first tested which of our face- and body-responsive ROls could decode body identity across viewpoint (Figure $5 b \&$ Table 5; for details of methods, see Section 2.9.2). From the identity recognition task, we could decode body identity across viewpoint significantly above chance-level $(1 / 3)$ in the body-responsive FBA $(36.2 \%$, $p=.0030)$ and face-responsive OFA (36.4\%, $p=.0035)$ and FFA $(35.6 \%, p=.024)$. We were not able to decode body identity across viewpoint from the body-responsive EBA or the face-responsive ATFA. From the viewpoint recognition task, we could decode body identity across viewpoint from the OFA $(37.1 \%, p<.001)$, but not from any other ROI.

To test whether there were significant differences in decoding performance across ROIs and Task, we performed a 5 (ROI) $\times 2$ (Task) repeated measures ANOVA. We found a significant main effect of $\mathrm{ROI}\left(F_{4,72}=4.40, p=.003, \eta_{p}{ }^{2}=0.20\right)$ and a significant interaction between $\mathrm{ROI}$ and Task $\left(F_{4,72}=3.84, p=.007, \eta_{p}{ }^{2}=0.18\right)$. Follow-up paired $t$-tests showed that during the identity recognition task decoding performance was higher in the OFA than in the ATFA and EBA (OFA \& ATFA: $t_{18}=2.19, p=.042$, Cohen's $d=0.50$; OFA \& EBA: $t_{18}=2.11, p=.049$, Cohen's $d=0.48$ ), higher in the FFA than the ATFA ( $t_{18}=3.17, p=.005$, Cohen's $d=0.73$ ), and higher in the FBA than in the EBA and ATFA (FBA \& EBA: $t_{18}=2.37, p=.029$, Cohen's $d=0.54$; FBA \& ATFA: $t_{18}=2.50, p=.022$, Cohen's $d=0.57$ ). During the viewpoint recognition task decoding performance was higher in the OFA than in all other ROIS (OFA \& FFA: $t_{18}=2.40, p=.027$, Cohen's $d=0.13$; OFA \& ATFA: $t_{18}=2.47$, $p=.024$, Cohen's $d=0.57$; OFA \& EBA: $t_{18}=2.41, p=.027$, Cohen's $d=0.55$; OFA \& FBA: $t_{18}=3.86, p=.001$, Cohen's $d=0.89$ ). Lastly, identity decoding performance was significantly higher during the identity recognition task compared to the viewpoint recognition task in the FBA ( $t_{18}=3.65, p=.002$, Cohen's $d=0.84$ ), but not in any other ROls.

We performed a whole-brain searchlight analysis to investigate if any other brain regions could decode body identity across viewpoint (Figure 6b). From both the identity and the viewpoint task data, we could decode body identity across viewpoint from a large cluster in occipital cortex (including the early visual cortex). Using fMRI data from the identity recognition task, we could additionally decode body identity across viewpoint from the middle frontal gyrus, right anterior temporal cortex, right superior parietal cortex, right medial superior frontal gyrus, right insula cortex, right rolandic operculum and the left motor cortex (due to participants' button presses). Using fMRI data from the viewpoint recognition task, we could additionally decode body identity across viewpoint from the left fusiform gyrus, right superior parietal cortex, left caudate nucleus, left cingulum and left postcentral gyrus. We additionally performed a paired $t$-test to investigate whether any regions showed significant differences in decoding performance during the two recognition tasks, but we did not identify any regions in this analysis.

\subsection{3 | Viewpoint-invariant body identity classification: ID2 versus ID3}

As we found higher BOLD responses to ID1 as compared to ID2 and ID3 in the OFA, FFA and FBA during the identity response task (Figure 3c), it is possible that our body identity decoding across viewpoint results in these regions was driven by differences in univariate activation. To explore whether purely multivariate pattern differences also contributed to body identity decoding across viewpoint in these 
TAB LE 5 Classification of body identity from patterns of BOLD responses in individual ROIs

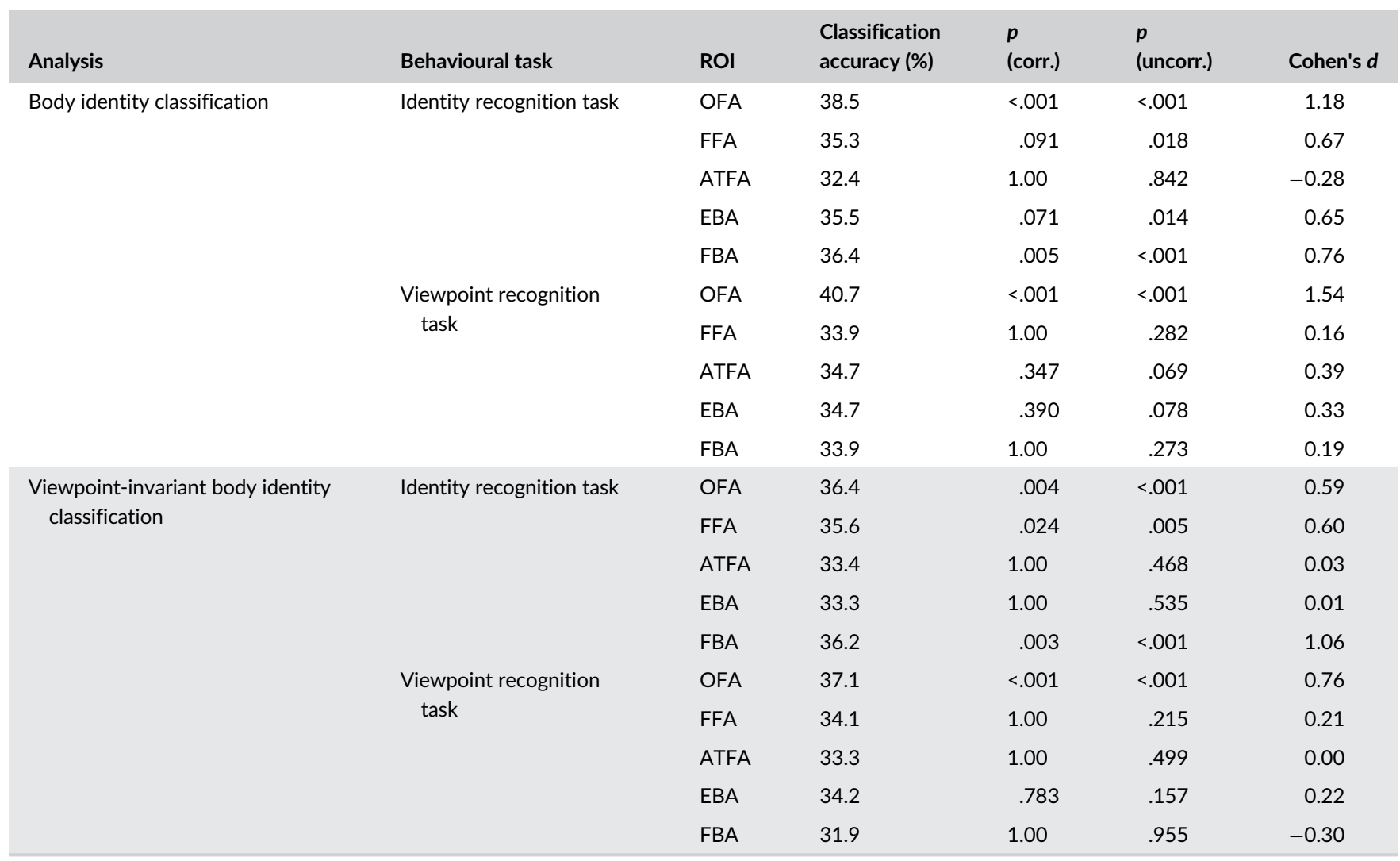

Note: Statistical significance was assessed using permutation tests, and $p$ values are shown before (uncorrected) and after (corrected) Bonferronicorrection for multiple comparisons ( $N=5$ ROIs).

regions, we performed an additional analysis to test if these regions would be able to classify only body identities ID2 and ID3 across viewpoint (Figure 5c). We performed the analysis using the same method as in Section 3.4.2, except that we only trained and tested the classifiers ability to distinguish between ID2 and ID3. We were able to decode body identity across viewpoint significantly above chance $(1 / 2)$ in the body-responsive FBA $(52.6 \%, p=.038$ Bonferroni corrected, Cohen's $d=0.54)$ but not in the face-responsive OFA (51.1\%, $p=.17$ uncorrected, Cohen's $d=0.28$ ) or FFA (51.5\%, 0.097 uncorrected, Cohen's $d=0.32$ ).

\section{5 | Identity classification across face and body stimuli}

Lastly, we performed multivoxel pattern analyses to investigate if any brain regions contain patterns of BOLD responses evoked by the identity of a person that could generalise across responses evoked by face and body stimuli (see Section 2.9.3 for method details), which would suggest the region encodes person identity in an abstract manner. We performed the analyses in face- and body-responsive ROls (Figure 7a \& Table 6) and in whole-brain searchlight analyses (Figure 7b).

Irrespective of using fMRI data from the identity recognition task or the viewpoint recognition task, we were unable to decode identity across BOLD responses evoked by faces and bodies higher than chance-level (1/3) in any of the ROls we tested (Figure 7a \& Table 6). We performed whole-brain searchlight analyses to investigate if any other brain regions could decode identity across BOLD responses evoked by faces and bodies. Using fMRI data from the identity recognition task (Figure $7 b$ ), we could decode identity from the early visual cortex (MNI: 10, -94, 2), a region in the right inferior occipital cortex (MNI: 40, -84, -4) overlapping with the mean location of the OFA, the right parahippocampal cortex ( $\mathrm{MNI}: 20,-4,-30$ ) and a region in the right superior parietal cortex (MNI: 16, $-56,60)$. We could also decode identity from the left motor cortex due to participants' button presses. In contrast, when using fMRI data from the viewpoint recognition task, we were not able to decode identity from any regions. To investigate whether any regions showed significant differences in decoding performance during the two recognition tasks we performed a paired $t$-test. We did not identify any regions showing significant differences in this analysis.

\subsection{Control analysis to test for similarities in low- level stimuli characteristics}

As our stimuli consisted of natural images, we performed a control analysis to evaluate if there were higher similarities between low-level stimulus characteristics for images within an identity compared to across identities that could have affected our decoding results. First, 
(a)

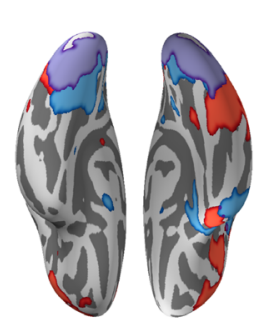

Body identity classification

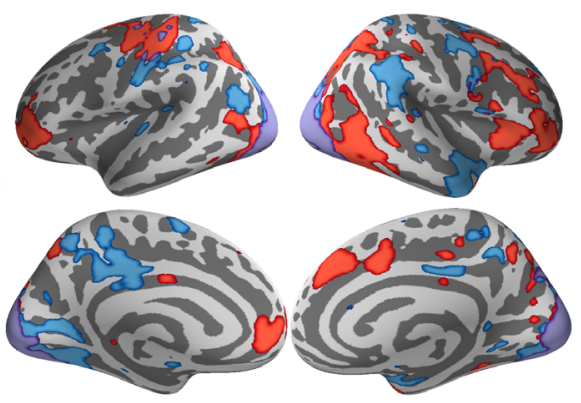

(b)

Viewpoint-invariant body identity classification
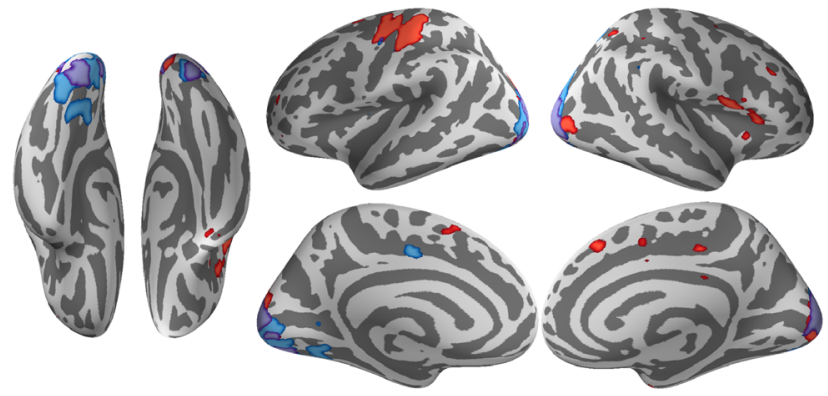

Identity recognition task

Viewpoint recognition task Conjunction

FIGURE 6 Classification (a) and viewpoint-invariant classification (b) of body identity in whole-brain searchlight analyses. Regions showing significant activity during the identity recognition task are shown in red, regions showing significant activity during the viewpoint recognition task are shown in blue and regions showing significant activity during both tasks (conjunction) are shown in purple. Significant regions were defined using a $p<.05$ FDR correction

we performed analyses separately for face and body images, to evaluate if there were similarities in low-level characteristics within images of a particular identity across different stimulus viewpoints. We performed correlations between red, green and blue pixel intensities between images of the same identity across viewpoint as well as between images differing in both identity and viewpoint. Then, we performed two-sample $t$-tests to investigate if there were significantly higher correlations between images of the same identity, compared to those of different identities.

For face images, we found no significant differences in correlations for red ( $\left.t_{24}=0.83, p=.417\right)$, green $\left(t_{24}=1.29, p=.208\right.$ ) or blue $\left(t_{24}=1.28, p=.214\right)$ pixel intensity values, suggesting that low-level characteristics were not more similar between face images of the same identity compared to different identities. However for bodies, we found significantly higher correlations between pixel intensity values for images within identity compared to across identity for all three colour values (red: $t_{24}=2.64, p=.0 .14$; green: $t_{24}=3.05$, (a)

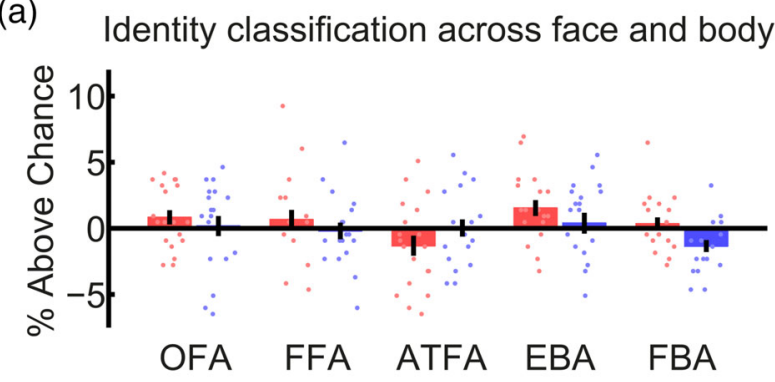

identity recognition task $\square$ viewpoint recognition task

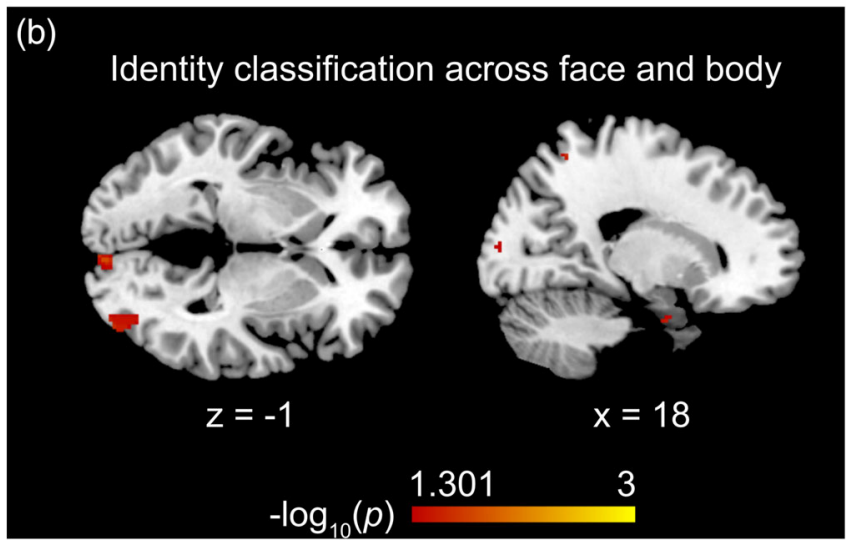

FIGURE 7 Classification of identity across BOLD responses evoked by faces and bodies. Panel (a) shows classification of identity above chance-level (1/3) across face and body stimuli in face- and body-responsive ROIs. Scatter points show classification accuracies for individual participants and error bars show \pm 1 SEM. Panel (b) shows classification of identity across face and body stimuli during the identity recognition task in a whole-brain searchlight analysis. The scale bar shows $-\log _{10}(p$ values) between $1.301(p=.05)$ and $3(p=.001)$, FDR corrected

$p=.006$; blue: $t_{24}=2.97, p=.007$ ). To further examine whether our results were due to these low-level image characteristics, we performed the same analysis including only ID2 and ID3, as we did for the additional fMRI analysis with these two identities in Section 3.4.3. We found no significant differences in red ( $\left.t_{10}=0.60, p=.564\right)$, green $\left(t_{10}=0.53, p=.609\right)$ or blue $\left(t_{10}=0.35, p=.737\right)$ pixel intensity values for images of these two identities. Note that we were still able to decode these two body identities based on BOLD responses in the FBA (Section 3.4.3).

Secondly, we performed an analysis to investigate if there were any similarities in low-level image characteristics between face and body images of the same identity. We performed correlations between red, green and blue pixel intensities of face and body images of the same identity and viewpoint, as well as between face and body images of different identities but same viewpoint. Then, we again performed two-sample $t$-tests to investigate if correlations were significantly higher between images of the same identity, compared to those of different identities. We found no significant differences in correlations for red $\left(t_{16}=-0.66, p=.517\right)$, green $\left(t_{16}=0.21\right.$, $p=.838)$ or blue $\left(t_{16}=0.01, p=.990\right)$ pixel intensity values, 
TAB LE 6 Classification of identity across BOLD responses evoked by face and body stimuli in ROls

\begin{tabular}{|c|c|c|c|c|c|}
\hline Behavioural task & ROI & Classification accuracy (\%) & $p$ (corr.) & $p$ (uncorr.) & Cohen's $d$ \\
\hline \multirow[t]{4}{*}{ Identity recognition task } & OFA & 34.2 & .917 & .183 & 0.35 \\
\hline & FFA & 34.0 & 1.00 & .231 & 0.20 \\
\hline & EBA & 34.9 & .210 & .042 & 0.58 \\
\hline & FBA & 33.7 & 1.00 & .339 & 0.16 \\
\hline \multirow{4}{*}{ Viewpoint recognition task } & FFA & 33.1 & 1.00 & .579 & -0.07 \\
\hline & ATFA & 33.4 & 1.00 & .484 & 0.01 \\
\hline & EBA & 33.7 & 1.00 & .332 & 0.11 \\
\hline & FBA & 32.0 & 1.00 & .935 & -0.67 \\
\hline
\end{tabular}

Note: Statistical significance was assessed using permutation tests, and $p$ values are shown before (uncorrected) and after (corrected) Bonferronicorrection for multiple comparisons ( $N=5 \mathrm{ROIs}$ ).

suggesting that low-level characteristics were not more similar within face and body images of the same identity compared to across identities.

\section{4 | DISCUSSION}

In this study, we investigated how face and body identities are encoded in the brain, and whether person identity is encoded in an abstract neural representation that generalises across face and body stimuli. Consistent with previous findings, we found that face identity could be decoded from BOLD responses in several distributed cortical regions (Anzellotti \& Caramazza, 2016). We found that body identity could also be decoded from BOLD responses in several distributed cortical regions, and in particular we found consistent decoding of body identity, including across viewpoint, from BOLD responses in the FBA, the right anterior temporal cortex, the middle frontal gyrus and the right insula cortex. We found we could decode identity in an abstract manner, across responses evoked by faces and bodies, from BOLD responses in the right parahippocampal cortex, right superior parietal cortex, right inferior occipital cortex and early visual cortex. These results provide new insights into how the brain encodes information about person identity.

\subsection{Neural coding of face identity}

Our ROI-based analysis showed that face identity can be decoded from BOLD responses in the face-responsive ATFA and bodyresponsive EBA. Our whole-brain searchlight analysis revealed a broader and more distributed brain network involved in encoding face identity, including the early visual cortex, inferior occipital cortex, fusiform gyrus, superior parietal cortex, superior temporal cortex, parahippocampal cortex, right middle frontal gyrus, right anterior cingulum, right medial superior frontal gyrus and left inferior frontal gyrus. These results are consistent with previous findings, which have shown that face identity can be decoded from a number of distributed brain regions, including the ATFA, FFA, OFA, superior intraparietal sulcus and right inferior frontal cortex (Anzellotti et al., 2014; Anzellotti \& Caramazza, 2016; Axelrod \& Yovel, 2015; Goesaert \& Op de Beeck, 2013; Guntupalli et al., 2017; Jeong \& Xu, 2016; Kriegeskorte et al., 2007; Natu et al., 2010; Nestor et al., 2011). Note that we could decode face identity only when participants attended to the identity of the stimuli, but not when they attended to the stimulus viewpoint. This suggests that face identity decoding in these regions may not be solely due to differences in visual features, as these were identical in both tasks (see Section 4.4 for a discussion of the behavioural task differences). However, we note that we did not identify a significant interaction between face identity decoding performance and recognition task in either our ROI or searchlight analyses.

Neither our ROI analysis nor our searchlight analysis showed above-chance decoding of face identity across viewpoint. Electrophysiological recordings in macaque monkeys have shown that neurons in the anterior face patches respond to face identity across viewpoint (Freiwald \& Tsao, 2010). Correspondingly, human neuroimaging studies have shown that face identity can be decoded across viewpoint from human face-responsive regions (Anzellotti et al., 2014; Guntupalli et al., 2017). One possibility for the discrepancy between these findings and our results is that people may need more extensive learning to develop viewpoint-invariant coding of face identity. In comparison to a training session of $30 \mathrm{~min}$ (Anzellotti et al., 2014) or with 360 trials (Guntupalli et al., 2017), our training (30 min for both face and body learning, with 135 face trials in total) might be insufficient for participants to establish a viewpoint-independent neural representation of face identity (despite high behavioural performance). Another possibility is that our sample size $(N=19)$ may have been too small to uncover significant patterns of face identity responses (Cremers, Wager, \& Yarkoni, 2017). Previous work has also demonstrated that in some cases fMRI MVPA can fail to decode identity, even when electrophysiological recordings show that viewpoint-invariant identity information is present in the underlying neurons (Dubois, de Berker, \& Tsao, 2015). 


\section{2 | Neural coding of body identity}

We could decode body identity from the body-responsive FBA and the face-responsive OFA and FFA in our ROI analyses, and from regions in the occipital cortex, fusiform gyrus, right anterior temporal cortex, superior parietal cortex, supramarginal gyrus, cingulum, precentral gyrus, caudate nucleus, inferior frontal gyrus, middle frontal gyrus, right insula cortex and right superior frontal gyrus in our searchlight analyses. Several of these regions have been shown to have higher responses to bodies of familiar people as compared to unfamiliar people (Hodzic et al., 2009). We could decode body identity from the OFA and occipital cortex during both recognition tasks, suggesting these regions may encode body identities using visual body features, and that their neural coding might not be influenced by top down factors such as attention. In contrast, decoding of body identity from the right anterior temporal cortex, inferior frontal gyrus, middle frontal gyrus and right insula was only possible when participants attended to identity.

In contrast to the decoding of face identity, our ROI analyses revealed viewpoint-tolerant encoding of body identity from BOLD responses in the FBA, OFA and FFA. Furthermore, viewpoint-tolerant coding of body identity was evident in more distributed regions in our searchlight analyses, including the occipital cortex, middle frontal gyrus, right anterior temporal cortex, right superior parietal cortex, right medial superior frontal gyrus, right insula cortex, right rolandic operculum, left caudate nucleus, left cingulum and left postcentral gyrus. Remarkably, the OFA and occipital cortex showed consistent above-chance decoding of body identity across viewpoints regardless of recognition tasks, suggesting that these regions may encode body identity using visual features that can generalise across different viewpoints. In a control analysis, we indeed found evidence of low-level characteristics that were more similar across body images of different viewpoints of the same identity as compared to different identities, which may be due to a difference in height of ID1 as compared to ID2 and ID3, as we found no difference between ID2 and ID3. In contrast to these findings in OFA and the occipital cortex, in the FBA viewpoint-invariant body identity decoding was significantly higher during the identity recognition task compared to the viewpoint recognition task, suggesting that body identity coding in the FBA was enhanced by participants' attention to identity. Furthermore, we could only decode body identity across viewpoint from the FFA, right anterior temporal cortex, middle frontal gyrus, right medial superior frontal gyrus, right insula cortex and right rolandic operculum when participants attended to identity. However, we note that we did not identify significant differences in decoding performance in these regions between the two recognition tasks.

Among the brain regions showing above chance decoding of body identities, FBA, FFA, OFA, right medial superior frontal gyrus and right insula also showed different univariate responses to the three body identities. For our ROIs, we performed a further analysis to disentangle whether the decoding of body identity in these regions was driven by purely univariate responses or also by multivariate differences in the pattern of BOLD responses. We found that we could decode body identity across viewpoint in the FBA between two identities that showed no difference in their univariate responses, demonstrating that multivariate patterns also contribute to body identity decoding across viewpoint in the FBA. Furthermore, in our control analysis for low-level visual characteristics, we found no evidence that low-level characteristics across different body viewpoints were more similar within identity than across identity for these two identities. Altogether, these results demonstrate a robust viewpoint-invariant neural encoding of body identity in the FBA. The FBA showed consistent decoding of body identity in two analyses (body identity decoding and viewpoint-invariant body identity decoding), and this decoding was not driven by low-level visual features.

Our finding that body identity can be decoded across viewpoint in the FBA and right anterior temporal cortex is consistent with the view that identity coding in the anterior temporal cortex is tolerant to viewpoint changes. Several previous studies have found viewpoint-invariant responses to face identity in the anterior temporal cortex (Anzellotti et al., 2014; Freiwald \& Tsao, 2010; Guntupalli et al., 2017). Similarly, classification of body identity across viewpoint and pose was higher in a more anterior body patch in macaque temporal cortex than a more posterior body patch (Kumar et al., 2019). Our results are consistent with these findings, suggesting that disentangling identity from viewpoint may be a general function of anterior temporal regions. Furthermore, our results also show a functional dissociation between neural coding of body identity in the FBA and the EBA, as viewpoint-invariant body identity decoding was significantly higher in the FBA than in the EBA. Furthermore, we were not able to decode body identity from the EBA in any of our analyses. Although a previous study found responses to body identity in both EBA and FBA using a repetition-suppression paradigm, feedback connectivity from the FBA to the EBA may have driven the body identity repetition suppression in the EBA in this study (Ewbank et al., 2011). Therefore, correspondingly to the coding of face identity along the posterior-anterior axis, encoding of body identity may also develop from viewpoint-sensitive in the EBA to viewpointinvariant in the FBA.

\section{3 | Neural coding of identity across face and body}

We could decode identity across BOLD responses evoked by face and body stimuli in the early visual cortex, the right inferior occipital cortex, the right parahippocampal cortex and the right superior parietal cortex, suggesting that these regions encode person identity in a manner abstracted from pure face- or body-related visual characteristics. Our ability to decode identity across the face and body in the early visual cortex may be due to feedback of identity information from high-level brain regions to the early visual cortex. Previous studies have demonstrated such feedback of high-level visual information to the early visual cortex (Bannert \& Bartels, 2013; Grassi, Zaretskaya, \& Bartels, 2017; Zaretskaya, Anstis, \& Bartels, 2013). Although we could not decode identity from our OFA ROI, we could decode identity from a region in the right inferior occipital cortex overlapping with the mean location of 
the right OFA. Together with the finding of viewpoint-invariant coding of face identity in the OFA (Anzellotti et al., 2014), this result suggests that inferior occipital cortex may contain some abstract encoding of person identity. We could also decode identity in an abstract manner in the right parahippocampal cortex, a region is known to be involved in memory and recollection (Eichenbaum, Yonelinas, \& Ranganath, 2007). Previous studies have shown an involvement of parahippocampal cortex in identity coding. Famous faces elicit stronger parahippocampal cortex activation as compared to unfamiliar faces (Bar, Aminoff, \& Ishai, 2008), and this region is also activated by recollection of contextual associations of faces and names (Kirwan \& Stark, 2004). Our results suggest that this region also integrates identity information from the face and body. Finally, the right superior parietal cortex also showed above-chance identity decoding across faces and bodies. Previous work has shown there is abstract coding of face and car identity in the parietal cortex (Jeong \& Xu, 2016). In combination, our identity decoding results across faces and bodies revealed a network of brain regions that respond to person identity in an abstract manner. These regions fall mostly outside of the standard face and body-responsive brain regions, suggesting that the integration of face and body identity occurs primarily outside of stimuli-selective brain regions.

\subsection{Effect of attention on neural coding of person identity}

We found that we could more frequently decode identity when participants attended to identity (performed the identity recognition task) than when they did not (performed the viewpoint recognition task). Furthermore, in the FBA we found significantly higher viewpoint-invariant body identity decoding performance during the identity recognition task compared to the viewpoint recognition task. In previous research, studies reporting successful decoding of face identity often used tasks where participants attended to identity (Anzellotti et al., 2014; Anzellotti \& Caramazza, 2016; Guntupalli et al., 2017; Jeong \& Xu, 2016; Nestor et al., 2011), whereas studies reporting unsuccessful decoding of face identity often used tasks that were unrelated to face recognition (Dubois et al., 2015; Ramírez, Cichy, Allefeld, \& Haynes, 2014). Recent studies have demonstrated that attention to face identity enhances neural responses to face identity (Dobs, Schultz, Bülthoff, \& Gardner, 2018; Gratton, Sreenivasan, Silver, \& D'Esposito, 2013). Our results, in combination with these previous studies, suggest that neural representations of face and body identity are enhanced by attention to identity, perhaps due to activation of identity-responsive neurons, and that this enhancement may be necessary to be able to decode person identity based on patterns of BOLD responses.

\section{5 | CONCLUSION}

We show, for the first time to our knowledge, that body identity can be decoded across viewpoint from fMRI activity in the bodyresponsive FBA, the right anterior temporal cortex, the middle frontal gyrus and the right insula cortex using MVPA. This result provides evidence that viewpoint-invariant identity coding may be a general function of more anterior regions of the human temporal cortex. Furthermore, we show that identity can be decoded in an abstract manner across fMRI activity evoked by faces and bodies in several brain regions previously associated with abstract identity coding. These results reveal how face and body identities are encoded, how this neural coding can generalise across viewpoints and how it is modulated by attention. Moreover, our results also shed light upon the neural substrates underlying the development of an abstract person identity representation.

\section{ACKNOWLEDGEMENTS}

This research was supported by the Max Planck Society, Germany. Open Access funding enabled and organized by Projekt DEAL.

\section{CONFLICT OF INTEREST}

The authors declare no potential conflict of interest.

\section{DATA AVAILABILITY STATEMENT}

Research data are not shared.

\section{ORCID}

Celia Foster (D) https://orcid.org/0000-0002-4507-5511

Mintao Zhao (D) https://orcid.org/0000-0003-0402-546X

Michael J. Black (D) https://orcid.org/0000-0001-6077-4540

Andreas Bartels (D) https://orcid.org/0000-0002-4939-7817

\section{REFERENCES}

Andrews, T. J., \& Ewbank, M. P. (2004). Distinct representations for facial identity and changeable aspects of faces in the human temporal lobe. Neurolmage, 23(3), 905-913. https://doi.org/10.1016/j.neuroimage. 2004.07.060

Anzellotti, S., \& Caramazza, A. (2016). From parts to identity: Invariance and sensitivity of face representations to different face halves. Cerebral Cortex, 26(5), 1900-1909. https://doi.org/10.1093/cercor/bhu337

Anzellotti, S., Fairhall, S. L., \& Caramazza, A. (2014). Decoding representations of face identity that are tolerant to rotation. Cerebral Cortex, 24(8), 1988-1995. https://doi.org/10.1093/cercor/bht046

Axelrod, V., \& Yovel, G. (2015). Successful decoding of famous faces in the fusiform face area. PLoS One, 10(2), 1-20. https://doi.org/10.1371/ journal.pone.0117126

Bannert, M. M., \& Bartels, A. (2013). Decoding the yellow of a gray banana. Current Biology, 23(22), 2268-2272. https://doi.org/10.1016/ j.cub.2013.09.016

Bar, M., Aminoff, E., \& Ishai, A. (2008). Famous faces activate contextual associations in the parahippocampal cortex. Cerebral Cortex, 18(6), 1233-1238. https://doi.org/10.1093/cercor/bhm170

Barton, J. J. S. (2008). Structure and function in acquired prosopagnosia: Lessons from a series of 10 patients with brain damage. Journal of Neuropsychology, 2(1), 197-225. https://doi.org/10.1348/174866407X214172

Brainard, D. H. (1997). The psychophysics toolbox. Spatial Vision, 10(4), 433-436. https://doi.org/10.1163/156856897X00357

Brandman, T., \& Yovel, G. (2016). Bodies are represented as wholes rather than their sum of parts in the occipital-temporal cortex. Cerebral Cortex, 26(2), 530-543. https://doi.org/10.1093/cercor/bhu205

Brooks, J. L. (2012). Counterbalancing for serial order carryover effects in experimental condition orders. Psychological Methods, 17(4), 600-614. https://doi.org/10.1037/a0029310 
Busigny, T., Van Belle, G., Jemel, B., Hosein, A., Joubert, S., \& Rossion, B. (2014). Face-specific impairment in holistic perception following focal lesion of the right anterior temporal lobe. Neuropsychologia, 56, 312333. https://doi.org/10.1016/j.neuropsychologia.2014.01.018

Cremers, H. R., Wager, T. D., \& Yarkoni, T. (2017). The relation between statistical power and inference in fMRI. PLoS One, 12(11), 1-20. https://doi.org/10.1371/journal.pone.0184923

Dobs, K., Bülthoff, I., \& Schultz, J. (2016). Identity information content depends on the type of facial movement. Scientific Reports, 6, 1-9. https://doi.org/10.1038/srep34301

Dobs, K., Schultz, J., Bülthoff, I., \& Gardner, J. L. (2018). Task-dependent enhancement of facial expression and identity representations in human cortex. Neurolmage, 172, 689-702. https://doi.org/10.1016/j. neuroimage.2018.02.013

Dubois, J., de Berker, A. O., \& Tsao, D. Y. (2015). Single-unit recordings in the macaque face patch system reveal limitations of fMRI MVPA. The Journal of Neuroscience, 35(6), 2791-2802. https://doi.org/10.1523/ JNEUROSCI.4037-14.2015

Eichenbaum, H., Yonelinas, A. P., \& Ranganath, C. (2007). The medial temporal lobe and recognition memory. Annual Review of Neuroscience, 30, 123-152. https://doi.org/10.1146/annurev.neuro.30.051606.094328

Ewbank, M. P., Lawson, R. P., Henson, R. N., Rowe, J. B., Passamonti, L., \& Calder, A. J. (2011). Changes in "top-down" connectivity underlie repetition suppression in the ventral visual pathway. The Journal of Neuroscience, 31(15), 5635-5642. https://doi.org/10.1523/JNEUROSCl. 5013-10.2011

Fisher, C., \& Freiwald, W. A. (2015). Whole-agent selectivity within the macaque face-processing system. Proceedings of the National Academy of Sciences, 112(47), 14717-14722. https://doi.org/10.1073/pnas. 1512378112

Foster, C., Zhao, M., Romero, J., Black, M. J., Mohler, B. J., Bartels, A., \& Bülthoff, I. (2019). Decoding subcategories of human bodies from both body- and face-responsive cortical regions. Neurolmage, 202, 1-13. https://doi.org/10.1016/j.neuroimage.2019.116085

Freiwald, W. A., \& Tsao, D. Y. (2010). Functional compartmentalization and viewpoint generalization within the macaque face-processing system. Science, 330, 845-851. https://doi.org/10.1126/science. 1194908

Gauthier, I., Tarr, M. J., Moylan, J., Skudlarski, P., Gore, J. C., \& Anderson, A. W. (2000). The fusiform "face area" is part of a network that processes faces at the individual level. Journal of Cognitive Neuroscience, 12(3), 495-504. https://doi.org/10.1162/089892900562165

Goesaert, E., \& Op de Beeck, H. P. (2013). Representations of facial identity information in the ventral visual stream investigated with multivoxel pattern analyses. The Journal of Neuroscience, 33(19), 8549-8558. https://doi.org/10.1523/JNEUROSCI.1829-12.2013

Grassi, P. R., Zaretskaya, N., \& Bartels, A. (2017). Scene segmentation in early visual cortex during suppression of ventral stream regions. Neurolmage, 146, 71-80. https://doi.org/10.1016/j.neuroimage.2016. 11.024

Gratton, C., Sreenivasan, K. K., Silver, M. A., \& D'Esposito, M. (2013). Attention selectively modifies the representation of individual faces in the human brain. The Journal of Neuroscience, 33(16), 6979-6989. https://doi.org/10.1523/JNEUROSCI.4142-12.2013

Grill-Spector, K., Knouf, N., \& Kanwisher, N. (2004). The fusiform face area subserves face perception, not generic within-category identification. Nature Neuroscience, 7(5), 555-562. https://doi.org/10.1038/nn1224

Guntupalli, J. S., Wheeler, K. G., \& Gobbini, M. I. (2017). Disentangling the representation of identity from head view along the human face processing pathway. Cerebral Cortex, 27(1), 46-53. https://doi.org/10. 1093/cercor/bhw344

Hadjikhani, N., \& de Gelder, B. (2002). Neural basis of prosopagnosia: An fMRI study. Human Brain Mapping, 16(3), 176-182. https://doi.org/10. 1002/hbm.10043
Hahn, C. A., \& O'Toole, A. J. (2017). Recognizing approaching walkers: Neural decoding of person familiarity in cortical areas responsive to faces, bodies, and biological motion. Neurolmage, 146, 859-868. https://doi.org/10.1016/j.neuroimage.2016.10.042

Hahn, C. A., O'Toole, A. J., \& Phillips, P. J. (2015). Dissecting the time course of person recognition in natural viewing environments. British Journal of Psychology, 107(1), 117-134. https://doi.org/10.1111/bjop.12125

Haxby, J. V., Hoffman, E. A., \& Gobbini, M. I. (2000). The distributed human neural system for face perception. Trends in Cognitive Sciences, 4(6), 223-233. https://doi.org/10.1016/S1364-6613(00)01482-0

Hebart, M. N., Görgen, K., \& Haynes, J.-D. (2015). The decoding toolbox (TDT): A versatile software package for multivariate analyses of functional imaging data. Frontiers in Neuroinformatics, 8, 1-18. https://doi. org/10.3389/fninf.2014.00088

Hodzic, A., Kaas, A., Muckli, L., Stirn, A., \& Singer, W. (2009). Distinct cortical networks for the detection and identification of human body. Neurolmage, 45(4), 1264-1271. https://doi.org/10.1016/j.neuroimage. 2009.01.027

Hoffman, E. A., \& Haxby, J. V. (2000). Distinct representations of eye gaze and identity in the distributed human neural system for face perception. Nature Neuroscience, 3(1), 80-84. https://doi.org/10.1038/71152

Jeong, S. K., \& Xu, Y. (2016). Behaviorally relevant abstract object identity representation in the human parietal cortex. The Journal of Neuroscience, 36(5), 1607-1619. https://doi.org/10.1523/JNEUROSCI.101615.2016

Jonas, J., Rossion, B., Brissart, H., Frismand, S., Jacques, C., Hossu, G., ... Maillard, L. (2015). Beyond the core face-processing network: Intracerebral stimulation of a face-selective area in the right anterior fusiform gyrus elicits transient prosopagnosia. Cortex, 72, 140-155. https://doi. org/10.1016/j.cortex.2015.05.026

Kirwan, C. B., \& Stark, C. E. L. (2004). Medial temporal lobe activation during encoding and retrieval of novel face-name pairs. Hippocampus, 14 (7), 919-930. https://doi.org/10.1002/hipo.20014

Kleiner, M., Brainard, D., \& Pelli, D. (2007). What's new in Psychtoolbox-3? In Perception 36 ECVP Abstract Supplement. Los Angeles, CA: Perception.

Kriegeskorte, N., Formisano, E., Sorger, B., \& Goebel, R. (2007). Individual faces elicit distinct response patterns in human anterior temporal cortex. Proceedings of the National Academy of Sciences, 104(51), 2060020605. https://doi.org/10.1073/pnas.0705654104

Kumar, S., Popivanov, I. D., \& Vogels, R. (2019). Transformation of visual representations across ventral stream body-selective patches. Cerebral Cortex, 29(1), 215-229. https://doi.org/10.1093/cercor/bhx320

Li, T., Bolkart, T., Black, M. J., Li, H., \& Romero, J. (2017). Learning a model of facial shape and expression from 4D scans. ACM Transactions on Graphics, 36(6), 1-17. https://doi.org/10.1145/3130800.3130813

Loffler, G., Yourganov, G., Wilkinson, F., \& Wilson, H. R. (2005). fMRI evidence for the neural representation of faces. Nature Neuroscience, 8 (10), 1386-1390. https://doi.org/10.1038/nn1538

Loper, M., Mahmood, N., Romero, J., Pons-Moll, G., \& Black, M. J. (2015). SMPL: A skinned multi-person linear model. ACM Transactions on Graphics (Proc. SIGGRAPH Asia), 34(6), 248:1-248:16. https://doi.org/ 10.1145/2816795.2818013

Nasr, S., \& Tootell, R. B. H. (2012). Role of fusiform and anterior temporal cortical areas in facial recognition. Neurolmage, 63(3), 1743-1753. https://doi.org/10.1016/j.neuroimage.2012.08.031

Natu, V. S., Jiang, F., Narvekar, A., Keshvari, S., Blanz, V., \& O'Toole, A. J. (2010). Dissociable neural patterns of facial identity across changes in viewpoint. Journal of Cognitive Neuroscience, 22(7), 1570-1582. https://doi.org/10.1162/jocn.2009.21312

Nestor, A., Plaut, D. C., \& Behrmann, M. (2011). Unraveling the distributed neural code of facial identity through spatiotemporal pattern analysis. Proceedings of the National Academy of Sciences of the United States of America, 108(24), 9998-10003. https://doi.org/10.1073/pnas.1102433108 
O'Toole, A. J., Phillips, P. J., Weimer, S., Roark, D. A., Ayyad, J., Barwick, R., \& Dunlop, J. (2011). Recognizing people from dynamic and static faces and bodies: Dissecting identity with a fusion approach. Vision Research, 51(1), 74-83. https://doi.org/10.1016/j.visres.2010. 09.035

O'Toole, A. J., Roark, D. A., \& Abdi, H. (2002). Recognizing moving faces: A psychological and neural synthesis. Trends in Cognitive Sciences, 6(6), 261-266. https://doi.org/10.1016/S1364-6613(02)01908-3

Pelli, D. G. (1997). The VideoToolbox software for visual psychophysics: Transforming numbers into movies. Spatial Vision, 10(4), 437-442. https://doi.org/10.1163/156856897X00366

Pitcher, D., Charles, L., Devlin, J. T., Walsh, V., \& Duchaine, B. (2009). Triple dissociation of faces, bodies, and objects in Extrastriate cortex. Current Biology, 19(4), 319-324. https://doi.org/10.1016/j.cub.2009. 01.007

Premereur, E., Taubert, J., Janssen, P., Vogels, R., \& Vanduffel, W. (2016). Effective connectivity reveals largely independent parallel networks of face and body patches. Current Biology, 26(24), 3269-3279. https:// doi.org/10.1016/j.cub.2016.09.059

Ramírez, F. M., Cichy, R. M., Allefeld, C., \& Haynes, J.-D. (2014). The neural code for face orientation in the human fusiform face area. The Journal of Neuroscience, 34(36), 12155-12167. https://doi.org/10.1523/ JNEUROSCI.3156-13.2014

Rice, A., Phillips, P. J., Natu, V., An, X., \& O'Toole, A. J. (2013). Unaware person recognition from the body when face identification fails. Psychological Science, 24(11), 2235-2243. https://doi.org/10.1177/ 0956797613492986

Rice, A., Phillips, P. J., \& O'Toole, A. (2013). The role of the face and body in unfamiliar person identification. Applied Cognitive Psychology, 27(6), 761-768. https://doi.org/10.1002/acp.2969

Robbins, R. A., \& Coltheart, M. (2012). The effects of inversion and familiarity on face versus body cues to person recognition. Journal of Experimental Psychology: Human Perception and Performance, 38(5), 10981104. https://doi.org/10.1037/a0028584
Rotshtein, P., Henson, R. N. A., Treves, A., Driver, J., \& Dolan, R. J. (2005). Morphing Marilyn into Maggie dissociates physical and identity face representations in the brain. Nature Neuroscience, 8(1), 107-113. https://doi.org/10.1038/nn1370

Schwarzlose, R. F., Baker, C. I., \& Kanwisher, N. (2005). Separate face and body selectivity on the fusiform gyrus. The Journal of Neuroscience, 25(47), 11055-11059. https://doi.org/10.1523/JNEUROSCI.262105.2005

Simhi, N., \& Yovel, G. (2016). The contribution of the body and motion to whole person recognition. Vision Research, 122, 12-20. https://doi. org/10.1016/j.visres.2016.02.003

Winston, J. S., Henson, R. N. A., Fine-Goulden, M. R., \& Dolan, R. J. (2004). fMRI-adaptation reveals dissociable neural representations of identity and expression in face perception. Journal of Neurophysiology, 92(3), 1830-1839. https://doi.org/10.1152/jn.00155.2004

Yovel, G., \& O'Toole, A. J. (2016). Recognizing people in motion. Trends in Cognitive Sciences, 20(5), 383-395. https://doi.org/10.1016/j.tics.2016.02.005

Zaretskaya, N., Anstis, S., \& Bartels, A. (2013). Parietal cortex mediates conscious perception of illusory gestalt. The Journal of Neuroscience, 33(2), 523-531. https://doi.org/10.1523/JNEUROSCI.2905-12.2013

\section{SUPPORTING INFORMATION}

Additional supporting information may be found online in the Supporting Information section at the end of this article.

How to cite this article: Foster, C., Zhao, M., Bolkart, T., Black, M. J., Bartels, A., \& Bülthoff, I. (2021). Separated and overlapping neural coding of face and body identity. Human Brain Mapping, 42(13), 4242-4260. https://doi.org/10.1002/ hbm.25544 